Pacific Journal of Mathematics

ON A NEW TYPE OF $L$-FUNCTION FOR ALGEBRAIC CURVES 


\title{
ON A NEW TYPE OF L-FUNCTION FOR ALGEBRAIC CURVES OVER FINITE FIELDS
}

\section{David Goss}

\begin{abstract}
Recently there has emerged a new theory of curves over a finite field. This theory is exciting in that it establishes previously unknown analogies between cyclotomic fields and function fields over a finite field. An extremely important aspect of this work is a new type of $L$-series for these function fields. These $L$-series bear quite remarkable and exciting similarities to classical $L$-series of number fields. The purpose of this paper is to describe these new $L$-series in detail.
\end{abstract}

\section{TABLE OF CONTENTS}

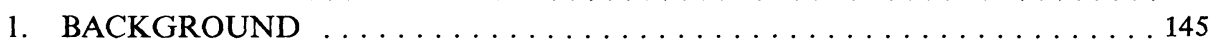

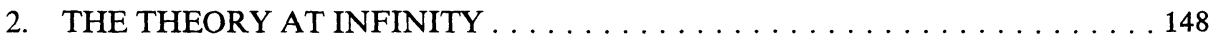

2.1 Basic concepts

2.2 A remark on the values of Dirichlet series

2.3 Definition of $\pi$-adic functions and their analytic continuation

2.4 Complements

3. DESCRIPTION OF VALUES AT POSITIVE INTEGER POWERS

3.1 The $\Gamma$-ideal

3.2 Special values of zeta functions at positive integral powers $\equiv 0(r-1)$

3.3 Special values of partial zeta-functions at positive integral powers

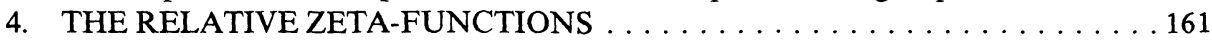

5. THE THEORY WHEN $s_{1}$ IS A NEGATIVE INTEGER $\ldots \ldots \ldots \ldots \ldots \ldots$

5.1 The algebraicity of $L$-series for $s_{1}=-i$

5.2 Some trivial zeros for zeta and $L$-functions

5.3 Some additional results when $A=\mathbf{F}_{r}[T]$

5.4 The $v$-adic theory and interpolation

5.5 A remark on functional equations

6. THE CONNECTION WITH DISTRIBUTION THEORY

6.1 Distribution and measures

6.2 The $\pi$-adic theory

6.3 Group theoretic interpretation of zeros

6.4 The $v$-adic theory

7. GALOIS GROUP INTERPRETATION VIA CLASS-FIELD THEORY . . . 179

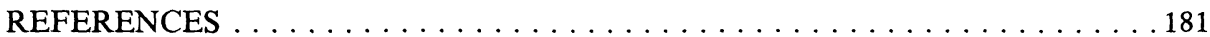

Our desire to study such functions arose out of the following fact (discovered by $\mathrm{L}$. Carlitz and independently, but much later, by the author): Let $i \in \mathbf{N}^{+}$. Then there exists a non-zero constant $\lambda$ such that the convergent $1 / T$-adic sum

$$
\lambda^{-i} \sum_{0 \neq \boldsymbol{\beta} \in \mathbf{F}_{r}[T]} \beta^{-i}, \quad i \equiv 0(r-1),
$$


actually is in $\mathbf{F}_{r}(T)$. One is immediately struck with the similarity to classical results on integer sums due to Euler. Carlitz also proved a vonStaudt result for these numbers that is close to the classical one, (see [7]).

The proof of these results involves the theory of elliptic modules ([1], [2], [3], [10], [11]) and their moduli. One is struck here with the analogy to cyclotomic fields. Because of these remarkable similarities, the author was led to search for the "meaning" along standard lines for these results. In particular, he followed classical ideas and introduced the continuous functions of ([6]) as the tools needed to accomplish this goal. We stress that this was a totally new type of undertaking and even involved a new type of analytic continuation.

The set-up that we use is more general than just polynomial rings: Let $C$ be a fixed smooth algebraic curve over $\mathbf{F}_{r}, r=p^{n}, p \in \mathbf{N}$. Let $k$ be the function field of $C$ and let $\infty$ be a fixed rational point. Finally, let $A$ be the Dedekind ring of those functions regular outside $\infty$. Then we use $A$ as the basic "integers" of our theory and define over objects with respect to $A$. (One instance is when $A=\mathbf{F}_{r}[T]$, as above.) We then study these objects at all the primes of $k$.

Historically, zeta-functions contain (at least) two types of information: The information contained in their special-values and the information contained in their zeroes. Unlike the classical theory of the Riemann zeta-function, our functions seem to have two distinct theories of specialvalues. One of these arises from the above facts, while the other arises when we give these functions their analytic continuation and its meaning is described in $\S 7$. As for the meaning of the zeroes of these functions there are some general themes running through this work that deserve special attention. The first is the "two-variable" property of our functions, (see [7]): Simply put, a two-variable function is a continuous function on a product of a characteristic- 0 space and a characteristic- $p$ space with analyticity in the finite characteristic space. All functions, at all primes, always end up being two-variable; an obvious though not well-understood, key to the theory.

The second concerns the general closeness of the theories at all primes of $k$ which was not apparent in ([6]). In fact, there is little difference between the theory at $\infty$ and any finite prime; the sole difference being the above mentioned results on power sums. For instance, at all primes, our functions are entire (see 2.1), at all primes they have Euler products, at all primes there are partial zeta-functions, etc. Thus their zero theory seems to be a curious amalgam: it contains aspects of both the classical $p$-adic and complex theories. This closeness is also borne out in the $\Gamma$-functions of ([7]). Finally, we point out that there are trivial zeroes at the negative integers for our functions in exact accordance with classical results. 
The third theme involves functional equations. It will become apparent to the reader that our functions are quite similar to classical $L$-series, but without their functional equations. So, for instance, one set of our special-values will contain a von Staudt type result while the other will contain congruences but no denominators, etc. As there does not appear to be any simple relationship between these values, we are thus presented with what appears to be a golden opportunity to obtain insight into functional equations. In fact, in 5.5 we will present some remarkable evidence for some sort of a functional equation, but of a radically new sort.

The fourth, and last, theme concerns the relationship between the zeroes of our functions at the various primes they are interpolated. In the case of the Artin zeta-function of a curve, this is trivial as the function is rational at all primes. But, for the Riemann zeta-function there is no apparent relationship between the zeroes of the complex and $p$-adic theories. On the other hand, our functions appear to possess a mechanism for passing between the various primes. Thus, they appear as a sort of "missing link" between the classical $L$-series of number fields and function fields.

In summary, we believe these new $L$-series are loaded with arithmetic information, (e.g., see §7). We also believe they offer a new and unique opportunity for insight into the classical functions. The situation is quite fluid and a new idea in any one of many areas will tell us much. We hope the reader will be inspired to probe deeper into the meaning of these functions and thereby discover the information they seem to so richly contain.

1. Background. As in the introduction, we have $\operatorname{Spec}(A)=C-\infty$, $k=$ the function field of $A$, and $K=k_{\infty}$, the completion at $\infty$. We let the symbol " $\mathfrak{B}$ " stand for finite primes of $A$ and " $\mathfrak{U}$ " to stand for $A$-fractional ideals. We let " $\hat{\mathfrak{U}}$ " denote the inverse fractional ideal. Associated to $\mathfrak{B}$, we have the additive valuation $v$. We let $A_{v}$ be the completion of $A$ at $v$, and $k_{v}$ the associated complete field. Finally, let $\theta_{\infty} \subseteq k$ be the ring of integers.

Let $\pi$ be a fixed uniformizer at $\infty$. We use the phrase " $\pi$-adic" to mean " $\infty$-adic". We also use " $v$-adic" to mean " $\$$-adic". Thus, $A$ is discrete $\pi$-adically but dense $v$-adically. We think of $A$ as being analogous to $\mathbf{Z}, A_{v}$ to $\mathbf{Z}_{p}$, etc.

For each $\mathfrak{A}$, we let $D(\mathfrak{A})$ be its degree as a finite divisor. Clearly, $D\left(\mathfrak{A}_{0} \mathfrak{A}_{1}\right)=D\left(\mathfrak{A}_{0}\right)+D\left(\mathfrak{A}_{1}\right)$. If $\mathfrak{A}=(a)$, we set $D(a)=D(\mathfrak{A})$. It is clear that $D(a)=$ order of pole of $a$ at $\infty$ \}. Finally, if $x \in K^{*}$, we set $D(x)=\{$ order of pole of $x$ at $\infty$.

Let $x \in K^{*}$. 
Definition. We say $x$ is monic (with respect to $\pi$ ) iff $x \pi^{D(x)} \in U_{1}=$ $\{1$-units at $\infty\}$. If $x$ is monic we set $\langle x\rangle=\pi^{D(x)} x$.

Any non-zero element differs from a monic by an element of $\mathbf{F}_{r}^{*}$. Further, if $x_{1}, x_{2}$ are monic with $D\left(x_{1}\right)=D\left(x_{2}\right)$, then $D\left(x_{1}-x_{2}\right)<$ $D\left(x_{1}\right)$. On the other hand, if $x$ is monic and $y$ arbitrary with $D(y)<D(x)$, then $x+y$ is still monic with $D(x+y)=D(x)$. Finally, if $x_{1}, x_{2}$ are monic, then $x_{1} x_{2}$ is also.

Notice that picking $\pi$ is the same as giving a splitting $K^{*}=\mathbf{Z} \times U$, where $U$ is the group of units at $\infty$. An interpretation in terms of class field theory is given in $\$ 7$.

As a convention, if a lower case English letter denotes an element of $k$, then it denotes a monic element. Further if $L$ is a field then we denote a fixed algebraic closure by $\bar{L}$ and we denote by $L_{\mathrm{ab}}$ the abelian closure of $L$ in $\bar{L}$.

Now let $L$ be an arbitrary field over $\mathbf{F}_{r}$ equipped with additive valuation $\boldsymbol{v}$. Let $W \subseteq L$ be a finite dimensional $\mathbf{F}_{r}$-vector space. The following lemma will be basic to the calculations that follow. Note the very strong estimates that it gives.

\subsection{LEMMA. Let $x \in L$. Then}

(1) $\sum_{w \in W}(x+w)^{i}=0$ for $0 \leq i<(r-1) \operatorname{dim}_{\mathbf{F}_{r}} W$.

(2) Assume $v(w)>0$ for all $w \in W$. Let

$$
W_{j}=\{w \in W \mid v(w) \geq j\} .
$$

Then,

$$
v\left(\sum_{w \in W} w^{i}\right) \geq(r-1)\left(\sum_{j \geq 1} \operatorname{dim} W_{j}\right) \quad \text { for all } i \in \mathbf{N} .
$$

Proof. Let $\left\{e_{1}, \ldots, e_{m}\right\}$ be a basis for $W$. By the multinomial theorem,

$$
\begin{aligned}
\sum_{w \in W}(x+w)^{i} & =\sum_{c_{1}, \ldots, c_{m} \in \mathbf{F}_{r}}\left(x+c_{1} e_{1}+\cdots+c_{m} e_{m}\right)^{i} \\
& =\sum_{j_{1} \cdots j_{m}} \frac{i ! x^{\left(l-j_{1} \cdots-j_{m}\right)}}{j_{1} ! \cdots j_{m} !\left(i-j_{1} \cdots-j_{m}\right) !} e_{1}^{j_{1}} \cdots e_{m}^{j_{m}} \boldsymbol{\delta}_{j_{1}} \cdots \delta_{j_{m}} ;
\end{aligned}
$$

where

$$
\delta_{j}=\sum_{c \in \mathbf{F}_{r}} c^{j}= \begin{cases}-1 & \text { if }(r-1) \mid j, j>0 \\ 0 & \text { otherwise }\end{cases}
$$

Therefore, 1 is immediate. 
Next, pick $\left\{e_{1}, \ldots, e_{m}\right\}$ so that $\left\{e_{1}, \ldots, e_{\operatorname{dim} W_{J}}\right\}$ is a basis for $W_{J}$, all $j$. Let $j_{0}$ be such that $W_{J_{0}+1}=\{0\}$, but $W_{J_{0}} \neq\{0\}$. By our choice of basis, any $w \in W$ can be written as

$$
W=P(w)+(w-P(w)),
$$

where $P$ is the projection onto $W_{J 0}$. Thus

$$
w^{l}=\sum_{d=0}^{i}(w-P(w))^{d} P(w)^{i-d}\left(\begin{array}{l}
i \\
d
\end{array}\right) .
$$

By applying (1) to $P(w) \in W_{J_{0}}$ and using induction, our sum has valuation $\geq(r-1) \sum_{j \geq 1} j \operatorname{dim}\left(W_{J} / W_{J+1}\right)=(r-1) \sum_{j \geq 1} \operatorname{dim} W_{j}$, and the lemma is established.

If $v(w)>0$, then it makes sense to raise $(1+w)$ to the $i$ th power for all $i \in \mathbf{Z}_{p}$.

1.3. Corollary. $v\left(\sum_{w \in W}(1+w)^{i}\right) \geq(r-1)\left(\sum_{j \geq 1} \operatorname{dim} W_{j}\right)$, all $i \in$ $\mathbf{Z}_{p}$.

Proof. Note that

$$
(1+w)^{l}=\sum_{l=0} w^{l}\left(\begin{array}{l}
i \\
l
\end{array}\right)
$$

Therefore, we can reduce to integral powers. The result follows from 1.2, as the estimate there is independent of the power involved.

Suppose that $\pi_{1}$ is another uniformizer at $\infty$. Then $\pi / \pi_{1}$ is a unit at $\infty$. Let $\pi_{1} / \pi \equiv \zeta(\pi), \zeta \in \mathbf{F}_{r}^{*}$. Then we have the obvious

1.4. LeMMA. $n$ is a monic with respect to $\pi$ iff $\zeta^{D(n)} n$ is monic with respect to $\pi_{1}$.

Thus, "monicity" carries only a small degree of ambiguity.

Next, we discuss some important abelian groups.

1.5. Definition. (a) Let $I \subseteq A$ be an ideal. We set $P(I)$ to be the group of ideals generated by all $(n)$, with $n \equiv 1(I), n \in A$. We let $P=P(A)$. We set $P^{0}(I)$ to be the group of all $(n)$ with $((n), I)=1$.

(b) We set $P\left(\pi^{m}\right)$ to be the group generated by all $(n)$ with $\langle n\rangle \equiv$ $1\left(\pi^{m}\right)$. We set $P\left(I \pi^{m}\right)$ to be the group generated by all $(n)$ with $\langle n\rangle \equiv 1\left(\pi^{m}\right)$ and $n \equiv 1(I)$. 

ideals.

1.6. Definition. (a) We set 9 to be the group of all $A$-fractional

(b) Let $C \subseteq A$ be an ideal. We set $\Phi(C)$ to be the group generated by all ideals prime to $C$.

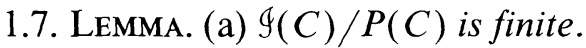

(b) $\mathscr{G}(C) / P\left(\pi^{m} C\right)$ is finite.

Proof. (a) is standard. To see (b) note that we have

$$
0 \rightarrow P(C) / P\left(\pi^{m} C\right) \rightarrow \frac{\Phi(C)}{P\left(\pi^{m} C\right)} \rightarrow \Phi(C) / P(C) \rightarrow 0
$$

and,

$$
0 \rightarrow P\left(\pi^{m} C\right) \rightarrow P(C) \rightarrow U_{1} / U_{1}(m) \rightarrow 0 ;
$$

where $U_{1}(m)=\left\{x \in K \mid x \equiv 1\left(\pi^{m}\right)\right\}$. The last arrow is surjective by the Riemann-Roch Theorem. This gives the result.

Note finally that we have

$$
0 \rightarrow P^{0}(C) / P\left(\pi^{m} C\right) \rightarrow \Phi(C) / P\left(\pi^{m} C\right) \rightarrow \Phi(C) / P^{0}(C) \rightarrow 0,
$$

with $9(C) / P^{0}(C) \cong \Phi / P$.

2. The theory at infinity. Our purpose here is to define the $L$-series and discuss their $\pi$-adic theory. Our method is quite in line with the classical. We first find a continuous space for which the notion " $n^{-s}$ ", $n \in A$, makes sense. Then our functions are defined by summation in the usual fashion. This summation converges on a "half-plane", but may be extended analytically to the whole space. This leads to the "two-variable" notion.

2.1. Basic concepts. Let $M$ denote the space of monic elements of $K$. Then $M=\pi^{\mathbf{Z}} \times U_{1} \cong \mathbf{Z} \times U_{1}$. Let $\chi$ be a continuous $K$-valued character of $M$. Then, it is clear that $\chi$ is determined by a pair $(a, E)$, consisting of an element $a \in K^{*}$ and an element $E \in \operatorname{End}_{\text {cont }}\left(U_{1}\right)$; where if $n=$ $\pi^{-D(n)}\langle n\rangle$, then we have

$$
\chi(n)=a^{D(n)} E(\langle n\rangle) .
$$

It is easy to see that $U_{1}$ is isomorphic to an infinite product of copies of $\mathbf{Z}_{p}$; where $\operatorname{End}_{\text {cont }}\left(U_{1}\right)$ is huge. In order to obtain something manageable, we replace the full space of endomorphisms with its subgroup $\mathbf{Z}_{p}=\{$ closure of integral powers $\}$. 
2.1.1. Definition. We set $S_{\infty}=K^{*} \times \mathbf{Z}_{p}$. We call $S_{\infty}$ the character space at infinity.

If $s=\left(s_{0}, s_{1}\right) \in S_{\infty}$, then $n^{s}=s_{0}^{D(n)}\langle n\rangle^{s_{1}}$, by definition.

Note that the integral powers appear in $S_{\infty}$ as $\left(\pi^{-i}, i\right)$. We refer to $\left(\pi^{-i}, i\right)$ as " $i$ ". These powers are discrete in the product topology on $S_{\infty}$. Further, $S_{\infty}$ forms an additive topological group in the obvious fashion.

We now want to make sense out of " $\mathfrak{U}^{-s}$ ", $s \in S_{\infty}$. Let $C \subseteq A$ be a fixed ideal.

2.1.2. Definition. We set $\hat{g}(C)=\lim \mathscr{G}(C) / P\left(\pi^{m} C\right)$. We set $\hat{g}=$ $\hat{g}(A)$. From the results of 1.7 , we see we have a map

$$
0 \rightarrow \underset{\overleftarrow{m}}{\lim _{\overleftarrow{m}}} P^{0}(C) / P\left(\pi^{m} C\right) \rightarrow \hat{g}(C) \rightarrow \mathscr{G}(C) / P^{0}(C) \rightarrow 0
$$

But,

$$
\lim _{\leftarrow} P^{0}(C) / P\left(\pi^{m} C\right) \underset{\leftarrow}{\sim} \lim _{\overleftarrow{m}} U_{1} / U_{1}(m)=U_{1} .
$$

Thus, we view $\hat{g}(C)$ as an extension of $U_{1}$ by $\mathscr{G}(C) / P^{0}(C) \cong$ Ideal class group of $A$.

2.1.3. Definition. Let $(\mathfrak{A}, C)=1$, we set

$$
\langle\mathfrak{A}\rangle=\{\text { Image } \mathfrak{A} \text { in } \hat{g}(C)\} \text {. }
$$

Let $\chi$ be a continuous $\bar{K}$-valued character on $\hat{g}$ (or more generally, a continuous map into $\mathrm{GL}(n, \bar{K}))$. We always assume that $\chi(\langle n\rangle)=\langle n\rangle^{s_{1}}$, for all $n$. (The reader can easily construct such objects.) By abusing notation, we write,

$$
\langle\mathfrak{A}\rangle^{s_{1}} \text { for } \chi(\langle\mathfrak{A}\rangle) \text {. }
$$

We do not, of course, specify $\chi$, but will always assume that one is chosen. The character $\chi$ is thus defined by its action on representatives of the classes of $G(C) / P^{0}(C)$. In order to keep matters from becoming unnecessarily confused, we work only with $\hat{g}$ in defining the $L$-series. The case when $C$ is arbitrary offers no extra difficulties.

2.1.4. Definition. Let $s=\left(s_{0}, s_{1}\right) \in S_{\infty}$. We set

$$
\mathfrak{U}^{s}=s_{0}^{D(\mathfrak{A})}\langle\mathfrak{U}\rangle^{s_{1}} \text {. }
$$

If $\mathfrak{A}=(n)$, then we see $\mathfrak{U}^{s}=n^{s}$ with the previous definition. 
If $\mathfrak{A}$ is a principal ideal, then we set $\theta(\mathfrak{A})$ to be its unique monic generator. Let $X=\left\{\mathfrak{U}_{0}, \ldots, \mathfrak{U}_{m}\right\}$ be a collection of representatives of $I / P$. If $\mathfrak{A} / \mathfrak{A}_{l}$ is principal, then we set

$$
\theta_{X}(\mathfrak{U})=\theta\left(\mathfrak{U} / \mathfrak{U}_{i}\right) .
$$

Thus, $\mathfrak{A}=\theta_{X}(\mathfrak{U}) \mathfrak{A}_{i}$, and $\mathfrak{U}^{s}=\theta_{X}(\mathfrak{A})^{s} \mathfrak{U}_{\imath}^{s}$.

Let $[L: K]<\infty$.

2.1.5. Definition. We call any series of the form

$$
\sum_{\mathfrak{U} \subseteq A} c(\mathfrak{A})^{-s}, \quad\{c(\mathfrak{A})\} \subseteq L,
$$

a formal Dirichlet series. Two such series are said to be equal iff all coefficients $\{c(\mathfrak{U})\}$ are equal.

From our axioms, we can sum $L(s)$ to obtain,

$$
\begin{aligned}
L(s) & =\sum_{i=0}^{m}\left(\sum_{a \in \hat{\mathfrak{A}}_{i}} c\left(a \mathfrak{U}_{i}\right) a^{-s}\right) \mathfrak{U}_{i}^{-s} \\
& =\sum_{i=0}^{m}\left(\sum_{a \in \hat{\mathfrak{A}}_{i}} c\left(a, \mathfrak{U}_{l}\right) a^{-s}\right) s_{0}^{-D\left(\mathfrak{A}_{l}\right)}\left\langle\mathfrak{U}_{i}\right\rangle^{-s_{1}} .
\end{aligned}
$$

2.1.6. Definition. We set $L\left(\mathfrak{U}_{l}, s\right)=\sum_{a \in \hat{\mathfrak{A}}_{l}} c\left(a \mathfrak{A}_{i}\right) a^{-s}$. Thus,

$$
L(s)=\sum_{i=0}^{m} L\left(\mathfrak{U}_{i}, s\right) \mathfrak{A}_{l}^{-s}=\sum_{i=0}^{m} L\left(\mathfrak{U}_{i}, s\right) s_{0}^{-D\left(\mathfrak{A}_{i}\right)}\left\langle\mathfrak{U}_{i}\right\rangle^{-s_{1}} .
$$

Further,

$$
L\left(\mathfrak{A}_{i}, s\right)=\sum_{\jmath>-\infty} s_{0}^{-j}\left(\sum_{\substack{D(a)=j \\ a \in \mathfrak{A}_{\imath}}} c\left(a \mathfrak{A}_{\imath}\right)\langle a\rangle^{-s_{1}}\right)
$$

2.1.7. Definition. We say that $L\left(\mathfrak{A}_{l}, s\right)$ has half-plane of convergence $D\left(s_{0}\right)>b$ iff for each fixed $s_{1} \in \mathbf{Z}_{p}$, and for each $i$, the power series for $L\left(\mathfrak{A}_{i},\left(s_{0}, s_{1}\right)\right)$ converges for all $s_{0}$ with $D\left(s_{0}\right)>b$ and the resulting function on $S_{\infty}$ is continuous. We say $L(s)$ is entire if we may let $b=-\infty$.

Note that the half-plane of convergence is obviously independent of $X$.

2.1.8. Definition. Fix $s_{1} \in \mathbf{Z}_{p}$. We say $L\left(\mathfrak{A}_{\imath},\left(s_{0}, s_{1}\right)\right)$ is a Laurentpolynomial iff it contains only finitely many powers of $s_{0}$ and $s_{0}^{-1}$. We say $L(s)$ is a Laurent-polynomial if $L\left(\mathfrak{A}_{l}, s\right)$ is one for all $i$. 
Finally, if $A=\mathbf{F}_{r}[T]$, we always set $X=\{A\}$ and do not necessarily

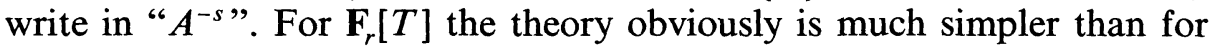
general $A$.

2.2. A remark on the values of Dirichlet Series. Let $L(s)$ be a Dirichlet series. Suppose $L(s)$ has half-plane of convergence $D\left(s_{0}\right) \geq b$. Then, for all $\left(s_{0}, s_{1}\right)$ with $D\left(s_{0}\right)>b$, the vanishing of

$$
\left|L\left(\mathfrak{U}_{i}, s\right)\right|_{\infty}
$$

is independent of $\mathfrak{A}_{i}$.

Thus, the zeroes of $L\left(\mathfrak{A}_{i}, s\right)$ are independent of the choice of $\mathfrak{U}_{i}$ in its ideal class.

Let $s \in S_{\infty}$.

2.2.1. Definition. (a) We say $L(s)=0$ unconditionally iff $L\left(\mathfrak{A}_{i}, s\right)=0$ all $i$.

(b) We say $L(s)=0$ conditionally iff $L(s)=0$ with the particular choice of character (or representation to $\mathrm{GL}(\mathfrak{A}, \bar{K})$ ).

Note that a implies $b$.

2.3. Definitions of the $\pi$-adic functions and their analytic continuations.

2.3.1. Definition. We call the function

$$
\prod_{\mathfrak{B} \text { prime }}\left(1-\mathfrak{B}^{-s}\right)^{-1}=\sum_{\mathfrak{A} \subseteq A} \mathfrak{U}^{-s},
$$

the zeta-function of $A$.

$\zeta(s)$ obviously converges for all $s$ with $D\left(s_{0}\right)>0$. Let $C \subseteq A$ be an ideal.

2.3.2. Definition. (a) Let $\delta \in \mathscr{G}(C) / P(C)$. We set

$$
\zeta(\delta, s)=\sum_{\substack{\mathcal{A} \in \delta \\ \mathfrak{U} \subseteq A}} \mathfrak{U}^{-s}
$$

It is called a partial zeta-function.

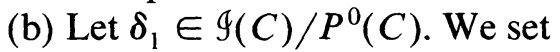

$$
\zeta\left(\delta_{1}, s\right)=\sum_{\delta \rightarrow \delta_{1}} \zeta(\delta, s) \quad(\text { " } \rightarrow \text { " means "projects"). }
$$

(Note that $\Sigma \zeta(\delta, s)=\zeta(s) \prod_{\mathfrak{P} \mid C}\left(1-\mathfrak{P}^{-s}\right)$.) 
(c) Let $f$ be a $\bar{K}$-valued function on $\mathscr{G}(C) / P(C)$. We set

$$
L(f, s)=\sum_{\substack{\mathfrak{A}, C)=1 \\ \mathfrak{U} \subseteq A}} f(\mathfrak{U}) \mathfrak{U}^{-s} .
$$

If $f=\chi$ is a character, then

$$
L(\chi, s)=\prod_{(\mathfrak{B}, C)=1}\left(1-\chi(\mathfrak{B}) \mathfrak{P}^{-s}\right)^{-1} .
$$
that

Let $X=\left\{\mathfrak{A}_{0}, \ldots, \mathfrak{A}_{m}\right\}$ and let $\delta$ be the ideal class of $\mathfrak{A}_{i}$. It is clear

$$
\zeta(\delta, s)=\zeta\left(\mathfrak{U}_{i}, s\right) \mathfrak{A}_{i}^{-s}, \quad \text { etc. }
$$

Then, the components of our functions are essentially partial zeta functions.

It is clear all these functions converge for $D\left(s_{0}\right)>0$. We now show that they are, in fact, entire.

Thus, let $\left\{\mathfrak{A}_{i}, C\right\}=1$, all $i$, and let $\delta=\beta+P(C), \beta \subseteq A$. If $(\mathfrak{A}, C)=1$, then $\mathfrak{A} \in \delta$ iff $\mathfrak{A} / \beta$ is principal and generated by a monic $\equiv 1(C)$. Thus,

$$
\zeta(\delta, s)=\sum_{i=0}^{m} \zeta\left(\delta, \mathfrak{A}_{i}, s\right) \mathfrak{A}_{i}^{-s}
$$

with,

$$
\zeta\left(\delta, \mathfrak{A}_{i}, s\right)=\sum_{n \in \mathfrak{A}_{i}} n^{-s}
$$

where $\left(n \mathfrak{A}_{i} / \beta\right)$ is principal and generated by a monic $\equiv 1(C)$. If $\mathfrak{A}_{i}$ and $\beta$ are not in the same class, then the condition is vacuous. If $\left\{\mathfrak{A}_{i}, \beta\right\}$ are in the same class, then our condition is the same as

$$
n \theta_{X}(\beta)^{-1} \equiv 1(C)
$$

or

$$
n \equiv \theta_{X}(\beta) \quad\left(\theta_{X}(\beta) C\right)
$$

As

$$
\left(\theta_{X}(\beta)\right)=\left(\beta / \mathfrak{Q}_{i}\right)
$$

we have

$$
\left(\theta_{X}(\beta) C\right) \subseteq \hat{\mathfrak{A}}_{i}
$$


Summarizing,

$$
\zeta\left(\delta, \mathfrak{A}_{i}, s\right)=\sum_{\substack{n \in \hat{\mathfrak{A}}_{i} \\ n \equiv \theta_{X}(\beta)\left(\boldsymbol{\theta}_{X}(\beta) C\right)}} n^{-s} .
$$

We remark that it is clear how to express $L(f, s)$ as a finite sum of partial zeta values. Further,

$$
\zeta(s)=\sum_{i=0}^{m}\left(\sum_{n \in \hat{\mathfrak{A}}_{i}} n^{-s}\right) \mathfrak{A}_{i}^{-s} .
$$

Thus,

$$
\zeta\left(\mathfrak{A}_{i}, s\right)=\sum_{\lambda \in \hat{\mathfrak{A}}_{i} /\left(\theta_{x}(\beta) C\right)}\left(\sum_{n \in \lambda} n^{-s}\right) .
$$

Therefore, to show our functions are entire, we are reduced to showing functions of the following form are entire: Let $\mathfrak{A}$ be a non-zero fractional ideal and let $I$ be a non-zero ideal. Let $\lambda \in \mathfrak{U} / I \mathfrak{U}$ and let

$$
L(s)=\sum_{\substack{n \in \mathfrak{R} \\ n \equiv \lambda(\mathfrak{I})}} n^{-s}
$$

Then we need to show $L(s)$ is entire.

\subsubsection{THEOREM. $L(s)$ is entire.}

Proof. It is clear that, upon possibly multiplying $L(s)$ by $a^{-s}$, some $a \in k$, we may assume $\mathfrak{A}$ is also integral.

Now,

$$
L(s)=\sum_{j \geq 0} s_{0}^{-j}\left(\sum_{\substack{n \in \mathfrak{A} \\ n=\lambda(I \mathscr{N}) \\ D(n)=j}}\langle n\rangle^{-s_{1}}\right) .
$$

Let $\langle n\rangle=1+w_{n}$. We show first that the set

$$
\begin{aligned}
& \left\{w_{n}\right\} \\
& n \in \mathfrak{A} \\
& n \equiv \lambda \\
& D(n)=j
\end{aligned}
$$

is a principal homogeneous space under a finite dimensional $\mathbf{F}_{r}$-vector space.

Let $n_{j}$ be a fixed element in our set. Let $n$ be an arbitrary such element. Then,

$$
n \equiv \lambda \equiv n_{j}(I \mathfrak{U})
$$


But $\left\{n-n_{j}\right\}$ is clearly a finite dimensional $\mathbf{F}_{r}$-vector space and

$$
w_{n}=w_{n_{j}}+\pi^{J}\left(n-n_{j}\right)=w_{n_{j}}+\left(w_{n}-w_{n_{j}}\right) .
$$

Consequently, $\left\{w_{n}-w_{n_{j}}\right\}$ is a finite dimensional $\mathbf{F}_{r}$-vector space. Thus, the set

$$
\begin{aligned}
& \left\{w_{n}\right\} \\
& n \in \mathfrak{N} \\
& n \equiv \lambda \\
& D(n)=J
\end{aligned}
$$

can be written as

$$
\left\{w_{n_{J}}+w\right\}
$$

with $w \in W(i)$ and $\operatorname{dim} W(i)<\infty$. Consequently,

$$
\begin{aligned}
& L(s)=\sum_{j \geq 0} s_{0}^{-J}\left(\sum_{\substack{D(n)=J \\
n \in I \mathscr{A}}} n^{-s_{1}}\right)=\sum_{j \geq 0} s_{0}^{-J}\left(\sum_{\substack{D(n)=j \\
n=\lambda \\
n \in \mathscr{N}}}\left(1+w_{n}\right)^{-s_{1}}\right) \\
& =\sum_{j \geq 0} s_{0}^{-J}\left(\sum_{w \in W(j)}\left(1+w /\left(1+w_{n_{j}}\right)\right)^{-s_{1}}\left(1+w_{n_{j}}\right)^{-s_{1}}\right) \\
& =\sum_{j \geq 0} s_{0}^{-j}\left(\left(1+w_{n}\right)^{-s_{1}} \sum_{w \in W(j)} \sum_{c=0}^{\infty}\left(\frac{w}{1+w_{n_{j}}}\right)^{c}\left(\begin{array}{r}
-s_{1} \\
c
\end{array}\right)\right) \\
& =\sum_{j \geq 0} s_{0}^{-j}\left(\sum_{w \in W(J)} \sum_{c=0}^{\infty} w^{c}\left(1+w_{n_{j}}\right)^{-\left(s_{1}+c\right)}\left(\begin{array}{r}
-s_{1} \\
c
\end{array}\right)\right) \text {. }
\end{aligned}
$$

Now the Riemann-Roch Theorem gives us the existence of elements $n \equiv \lambda(I \mathscr{U})$ with $D(n)=j, j \gg 0$. Indeed, for $j \gg 0$ these are of the form $\lambda+h, h \in I \mathfrak{A}, D(h)=j$. As $j=D(h)$ grows, we can produce elements $w_{n}$ with arbitrary order of zero at $\infty$, between 1 and $\mathbf{N}(j) \in \mathbf{N}$. Further, we may let $\mathbf{N}(j+1)=\mathbf{N}(j)+1$. So the estimate of 1.2 tells us that the coefficient of $s_{0}^{-j}$ is divisible by $\pi^{f(j)}$ with $f(j)$ quadratic in $j$. The result easily follows.

\subsubsection{COROLlary. The functions of 2.3.1, 2.3.2 are entire.}

We now define, for future use, $\pi$-adic functions that have no known classical counterpart. They can be thought of as partial zeta-functions at $\infty$, and, in the case $m=1$, they reduce to those previously defined.

We use the notation of 1.5 . Let $\mathfrak{B}$ be an ideal and let $\delta$ be a class of

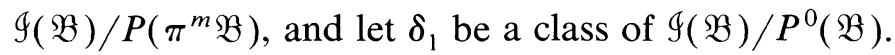


2.3.5. Definition. (a) We set

$$
\zeta(\delta, s)=\sum_{\substack{\mathscr{A} \subset A \\ \mathscr{U} \in \boldsymbol{\delta}}} \mathfrak{U}^{-s}
$$

(b) We set $\zeta\left(\delta_{1}, s\right)=\Sigma_{\delta \rightarrow \delta_{1}} \zeta(\delta, s)$.

(c) Let $f$ be a $K$-valued function on $\mathscr{G}(\mathfrak{B}) / P\left(\pi^{m} \mathfrak{B}\right)$. We set

$$
L(f, s)=\sum_{\delta} f(\delta) \zeta(\delta, s)
$$

Note that

$$
\sum_{\delta} \zeta(\delta, s)=\left(\prod_{\mathfrak{B} \mid \mathfrak{B}}\left(1-\mathfrak{B}^{-s}\right)\right) \zeta(s) .
$$

2.3.6. THEOREM. The functions of 2.3.5 are entire.

Proof. As before, we reduce to handling functions of the form

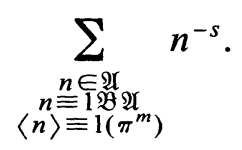

Let $\lambda \in \mathfrak{B} \mathfrak{A}$ have degree $<j-m$ and assume that $j>m$. Let $n \in \mathfrak{A}$ have degree $j$. Then

$$
D(n+\lambda)=D(n), \quad n+\lambda \equiv n(\mathfrak{B} \mathfrak{U}),
$$

and

$$
\langle n+\lambda\rangle=\langle n\rangle+\pi^{m}\left(\pi^{j-m} \lambda\right) \equiv\langle n\rangle\left(\pi^{m}\right) .
$$

Conversely, if $D\left(n_{1}\right)=D\left(n_{2}\right)=j, \quad n_{1} \equiv n_{2}(\mathfrak{B} \mathfrak{U}), \quad$ and $\left\langle n_{1}\right\rangle \equiv$ $\left\langle n_{2}\right\rangle\left(\pi^{m}\right)$, then $n_{1}-n_{2} \in \mathfrak{B} \mathfrak{A}$ and $\pi^{j}\left(n_{1}-n_{2}\right) \in\left(\pi^{m}\right)$. Thus, $n_{1}-$ $n_{2}=\lambda$ is as above.

With these observations, we can proceed as before to the results.

2.4. Complements. (1) Let $s_{1} \in \mathbf{Z}_{p}$ and let $L(s)$ be a component of one of the functions discussed in the previous chapter. As $L\left(s_{0}, s_{1}\right)$ is entire, it has a factorization

$$
L\left(s_{0}, s_{1}\right)=c\left(s_{1}\right) s_{0}^{k\left(s_{1}\right)} \prod_{\alpha \in Z\left(s_{1}\right)}\left(1-s_{0}^{-1} / \alpha\right),
$$

where $c\left(s_{1}\right)$ is a constant, $k\left(s_{1}\right) \in \mathbf{Z}$, and $Z\left(s_{1}\right)$ is the set of zeroes of $L\left(s_{0}, s_{1}\right)$ counted with multiplicity. We remark that a very similar expression is also known for classical zeta-functions. On the other hand, whereas classical functions are essentially entire of order one, ours correspond to entire functions of order zero. 
In the case of the zeta-function, it would be very interesting to see if there are any relations between the zero sets of the different components. We present a result in this direction in 5.2.

(2) It is interesting to compare the situation described above with the situation of the $q$-expansions, as described in ([5]). Indeed, the $q$-expansions of Eisenstein series involve sums for each coefficient $q^{i}, i \in \mathbf{N}$. Further, for each non-zero $\beta$ (a "normalizing factor") the coefficient of $q_{\infty}^{i}$ is multiplied by $\beta^{i}$. This should be contrasted with the fact that our $L$-series appear as sums of $s_{0}^{-i}, i>-\infty$, and each coefficient of $s_{0}^{-j}$ involves sums not unlike those associated to the Eisenstein series.

(3) Finally, let $i \in \mathbf{Z}$. As a corollary of our results, we see sums of the form

$$
\sum_{j \geq 0} s_{0}^{-j}\left(\sum_{\substack{D(n)=j \\ n \in \hat{\mathfrak{A}}}} n^{i}\right)
$$

are entire as a function of $s_{0}^{-1}$. Indeed, this particular example is $\zeta\left(\mathfrak{U},\left(s_{0} / \pi^{i}, i\right)\right)$.

3. Description of values at positive integer powers. For completeness, we now summarize the results on values of $\zeta(s)$ as described in ([7]). However, using the results of ([11]), we can simplify the exposition given there.

\subsection{The $\Gamma$-ideal. Let $x$ be a transcendental element.}

3.1.1. Definition. (1) We set $[i](x)=x^{r^{i}}-x, i \in \mathbf{N}^{+}$.

(2) We set $D_{0}(x)=1$ and $D_{i}(x)=[i][i-1]^{r} \cdots[1]^{r^{-1}}(x)$, for $i>0$.

3.1.2. Definition. (1) Let $i \in \mathbf{N}$ and let $\sum_{b=0}^{v} \alpha_{b} r^{b}$, be its $r$-adic expansion. For $a \in A$, we set

$$
\Gamma(a, i)=D_{0}(a)^{\alpha_{0}} \cdots D_{v}^{\alpha_{v}}(a) .
$$

(2) We set

$$
\Gamma_{i}=(\Gamma(a, i))_{a \in A} .
$$

We call $\Gamma_{i}$, the $i$ th $\Gamma$-ideal.

3.1.3. Proposition. (Sinnott). Let $\mathfrak{B} \subseteq A$ be a prime and $D(\mathfrak{B})$ its degree. Set $\alpha_{\mathfrak{P}}(i)=\Sigma_{e \geq 1}\left[i / r^{e D(\mathfrak{P})}\right]$ (where [ ] means greatest integer). Then,

$$
\Gamma_{i}=\prod_{\mathfrak{P} \text { prime }} \mathfrak{P}^{\alpha_{\mathfrak{B}}(i)}
$$


3.1.4. Corollary. Let $A=\mathbf{F}_{r}[T]$, then

$$
\Gamma_{i}=(\Gamma(T+\zeta, i)), \quad \zeta \in \mathbf{F}_{r} .
$$

The $\Gamma$-ideals are our "factorial".

3.2. Special values of zeta-functions at positive integral powers $\equiv 0$ $(r-1)$. Let $F$ be a field over $k$ contained in $\bar{K}$.

\subsubsection{Definition. Let}

$$
L(s)=\sum_{J=0}^{m} L\left(\mathfrak{A}_{J}, s\right) \mathfrak{A}_{i}^{-s}=\sum_{j=0}^{m} L\left(\mathfrak{U}_{J}, s\right) s_{0}^{-D\left(\mathfrak{A}_{J}\right)}\left\langle\mathfrak{U}_{j}\right\rangle^{-s_{1}} .
$$

Suppose $L(s)$ has half-plane of convergence $D\left(s_{0}\right)>b$. Let $i=\left(\pi^{-i}, i\right)>$ $b$ and let $\lambda \in \bar{K}^{*}$. We say

$$
\lambda^{-\imath} L(i) \in F
$$

iff

$$
\lambda^{-i} L\left(\mathfrak{U}_{J}, i\right)=\left(\frac{\pi^{-D\left(\mathfrak{A}_{J}\right)}}{\lambda}\right)^{l}\left(L\left(\left(\mathfrak{U}_{j}, i\right)\left(\pi^{-i}\right)^{-D\left(\mathfrak{A}_{\iota}\right)}\right)\right)
$$

is in $F$ for all $j$. (Note that this concept is independent of our choice of $\left\{\mathfrak{U}_{l}\right\}$.)

Let $M_{1}$ be the coarse moduli space of elliptic modules of rank 1 . Thus, $M_{1}$ is the spectrum of the ring of $A$-integers in the Hilbert class-field, $H$, which is totally-split ("totally-real") at $\infty$. In ([11]), Hayes shows the existence of a rank one elliptic module, $(\varphi)$, with coefficients in the ring $\vartheta_{H}$ of $A$-integers of $H$.

Let $\mathfrak{A}_{0} \lambda$ be the rank-one lattice associated to $(\varphi)$, where $\mathfrak{U}_{0}$ is a fractional-ideal. By applying $\operatorname{Gal}\left(M_{1} / A\right)$ to $(\varphi)$, we can construct over $\vartheta_{H}$, elliptic modules whose lattice is $\mathfrak{A}_{i} \lambda$, where $\left\{\mathfrak{A}_{i}\right\}$ runs through representations of the distinct ideal classes. We use the collection $X=$ $\left\{\mathfrak{U}_{0}, \ldots, \mathfrak{U}_{m}\right\}$ in our decomposition of $\zeta(s)$.

Now let $i \in \mathbf{N}^{+}$, with $i \equiv 0(r-1)$. Then we have

\subsubsection{THEOREM. $\lambda^{-i} \zeta(i) \in H$.}

Proof. We just sketch the procedure for calculation. Let $\sigma \in$ $\operatorname{Gal}\left(M_{1} / A\right)$ and let $\left(\varphi^{\sigma}\right)$ be $(\sigma(\varphi))$. Further, let $L=\mathfrak{A}_{J} \lambda$ be the lattice associated to $\left(\varphi^{\sigma}\right)$.

Let $e(z)=z+\sum_{i=0}^{\infty} c_{i} z^{r^{i}}$, be a formal power series with unknown coefficients, and let $a \in A$. We assume that for $a \in A-\mathbf{F}_{r}, e(z)$ satisfies the composition functional equation.

$$
e(a z)=\varphi^{\sigma}(a)(e(z)) .
$$


We may therefore find $\left\{c_{i}\right\}$ by induction. It is a theorem that $e(z)$ is everywhere convergent and that

$$
e(z)=z \prod_{0 \neq \alpha \in L}(1-z / \alpha) .
$$

As $e^{\prime}(z) \equiv 1, z e^{\prime}(z) / e(z)=z / e(z)=z \Sigma_{\alpha \in L}(z+\alpha)^{-1}$; whence the non-zero Taylor coefficients of $z / e(z)$ are of the form

$$
-\lambda^{-i \zeta}\left(\mathfrak{U}_{j}, i\right)
$$

for $i \equiv 0(r-1) ; 0$ otherwise. On the other hand, these can be computed directly by long division. The result is now easy.

We call $\lambda$ "the period of $(\varphi)$ ".

3.2.3. Question. For $A=\mathbf{F}_{r}[T]$, it is known that $\lambda$ is transcendental, see $([12])$. Is it so in general?

It is known that, as $\sigma \in \operatorname{Gal}\left(M_{1} / A\right)$ varies, the values

$$
\lambda^{-i} \zeta\left(\mathfrak{U}_{j}, i\right)
$$

$i \equiv 0(r-1)$, are conjugate. They therefore generate a Galois stable fractional ideal over $\theta_{H}$. It, therefore, descends to an $A$-fractional ideal $\subseteq k$, that we denote

$$
\lambda^{-i} \widehat{\zeta(i)} \text {. }
$$

3.2.4. Definition. We call $B_{i}=\Gamma_{i} \lambda^{-i} \widehat{\zeta(i)}, i \equiv 0(r-1), i \in \mathbf{N}^{+}, B_{i}$ $=(0), i \neq 0(r-1)$, the $i$ th Bernoulli-Carlitz fractional ideal.

We now state the basic result concerning the denominator of $B_{i}$. For a proof, see ([7]). In the case $A=\mathbf{F}_{r}[T]$, it is due to L. Carlitz, as are the basic ideas of proof.

Recall $r=p^{n}$.

3.2.5. TheOREM. $(r \neq 2)$. Let $i \equiv 0(r-1)$ and let $\sum_{b=0}^{v} \alpha_{b} r^{b}$, be its $p$-adic expansion. We have two conditions on $i$,

$$
\sum \alpha_{b} /((p-1) n)=h \in \mathbf{Z}
$$

and

$$
\left(r^{h}-1\right) \mid i \text {. }
$$

If $i$ satisfies both these conditions, then the denominator of $B_{i}=$ $\Pi_{\mathfrak{P} \text { prime, } D(\mathfrak{P})=h} \mathfrak{P}$. If not, then $B_{\imath} \subseteq A$. 
3.2.6. THEOREM. $(r=2)$. We retain the set-up of 3.2.5. If $i$ satisfies the conditions (1), (2) of 3.2 .5 and $h \neq 2$, then the denominator of $B_{i}$ is $\prod_{\mathfrak{B} \text { prime, } D(\mathfrak{P})=h} \mathfrak{B}$.

If $h=2, i$ even, then the denominator

$$
\prod_{\substack{D(\mathfrak{B})=2 \\ \mathfrak{P} \text { prime }}} \mathfrak{B} .
$$

If $h=2, i$ odd, then the denominator is

$$
\prod_{\substack{D(\mathfrak{P})=1 \text { or } 2 \\ \mathfrak{P} \text { prime }}} \mathfrak{P} .
$$

If $i$ doesn't satisfy the conditions, then for $i$ even

$$
B_{\imath} \subseteq A,
$$

while for $i$ odd, the denominator is

$$
\prod_{\substack{\mathfrak{B} \text { prime } \\ D(\mathfrak{P})=1}} \mathfrak{R} .
$$

3.2.6. Remarks. (1) The $\Gamma$-values may be interpolated Morita-style at all primes of $k$ to two-variable functions. For the details, we refer the reader to ([7]). (In keeping with the present work, it seems natural to extend the definition of ([7]) at $\infty$ as follows: Let $a \in k^{*}$, then we set for $i \in \mathbf{Z}_{p}$,

$$
\Gamma_{\infty}(a, i)=\Gamma_{\infty}(\langle a\rangle, i),
$$

where the element on the right is defined in ([7]). This definition reflects the fact that at $\infty$ our functions should be defined via $\pi$.) It seems clear that these $\Gamma$-functions are intimately related to the $L$-series, see 5.5.

(2) The proof of 3.2.5 and 3.2.6 is reminiscent of the proof of Stickelberger's Theorem on Gauss sums.

3.3. Special values of partial zeta-functions at positive integral powers. Let $0 \neq \mathfrak{A}$ be a fractional ideal, of $k, I$ an integral ideal and $\alpha \in \mathfrak{A}-I \mathfrak{A}$. By 2.3 , it is clear that we may restrict ourselves to studying functions of the form

$$
\sum_{\substack{n \in \mathfrak{A} \\ n \equiv \alpha(I \mathscr{N})}} n^{-s}
$$

For now, let the above sum be denoted $L(\alpha, s)$. 
3.3.1. Proposition. Let $i \in \mathbf{N}^{+}$. Then,

$$
\sum_{\beta \in \mathbf{F}_{r}^{*}} \beta^{i} L(\beta \alpha, i)=\sum_{\substack{\text { all } \delta \in \mathcal{Q} \\ \delta \equiv \alpha(I \mathscr{R})}} \delta^{-i} .
$$

Proof. We assume that $\alpha$ is chosen of least degree in its residue class. Let $h \in I \mathfrak{A}$; we have three cases:

(1) $D(h)>D(\alpha)$. In this case, $\alpha+h$ is monic, while $\alpha+\zeta h$ is not for $\zeta \in \mathbf{F}_{r}^{*}-\{1\}$. Thus, the sum on the left contributes

$$
\sum_{\beta \in \mathbf{F}_{r}^{*}}\left(\alpha+\beta^{-1} h\right)^{-1}
$$

so the result is obvious in this case.

(2) $D(h)<D(\alpha)$. In this case $\alpha+\zeta h$ is monic iff $\alpha$ is. The left-hand sum has a non-zero contribution only for those $\beta$ with $\beta \alpha$ monic; in which case it contributes,

$$
\begin{cases}\sum_{\zeta \in \mathbf{F}_{r}^{*}}\left(\alpha+\beta^{-1} \zeta h\right)^{-1} & \text { if } h \neq 0, \\ \alpha & \text { if } h=0 ;\end{cases}
$$

again the result is obvious.

(3) $D(h)=D(\alpha)$. In this case, for some $\zeta \in \mathbf{F}_{r}^{*}, D(\alpha-\zeta h)<D(\alpha)$. Thus we are back in case (1).

Now let $(\psi)$ be an elliptic module of rank one defined over some affine piece of $\theta_{H}$, as previously discussed, chosen so that its lattice is $c I \mathfrak{A} \lambda$ for some $c \in k^{*}$, and period $\lambda$.

3.3.2. TheOREM. $\lambda^{-i}\left(\Sigma_{\beta \in \mathbf{F}_{r}^{*}} \beta^{i} L(\beta \alpha, i)\right) \in k_{\mathrm{ab}}$, for all $i \in \mathbf{N}^{+}$.

Proof. As before, we present a sketch. Also, as before, we let

$$
e(z)=z \prod_{0 \neq \delta \in c I \mathscr{A} \lambda}(1-z / \lambda \delta) .
$$

Then, $e(z)$ has the functional equation,

$$
e(a z)=\psi(a)(e(z)) \text {, }
$$

for all $a \in A$.

Let $0 \neq i \in I$. It is clear that $i \alpha \in I \mathfrak{A}$. Thus, from above, we see

$$
e(\lambda \alpha)
$$

is a root of $\psi(i)(x)=0$, i.e., it is an $I$-division point. Further,

$$
\frac{1}{e(z+\lambda \alpha)}=\frac{1}{e(z)+e(\lambda \alpha)}=\sum_{\delta}(z+\lambda(\alpha+\delta))^{-1} .
$$


Thus, the Taylor coefficients are, on the one hand, in $k_{\mathrm{ab}}$, while, on the other hand, of the form $\pm(\lambda c)^{-i} \Sigma_{\delta \in I \mathfrak{A}}(\alpha+\beta)^{-i}$. The previous proposition now finishes the proof.

3.3.3. Remark. The proof of 3.3 .2 shows

$$
\frac{1}{e(\lambda \alpha)}=\lambda^{-1} \sum_{\delta \in I \mathfrak{A}}(\alpha+\delta)^{-1}=\lambda^{-1} \sum_{\beta \in \mathbf{F}_{r}^{*}} \beta L(\beta \alpha, 1) .
$$

Now set $I=\mathfrak{P}^{n}$, and let $\mathfrak{A}=\mathfrak{B}^{-m} \mathfrak{U}_{1}$. Further, let $\alpha_{0}, \alpha_{1} \in \mathfrak{U}-$ $\mathfrak{P}^{-(m-1)} \mathfrak{A}_{1}$. Then we have just seen

$$
\frac{e\left(\lambda \alpha_{1}\right)}{e\left(\lambda \alpha_{0}\right)}=\frac{\sum_{\beta \in \mathbf{F}_{r}^{*}} \beta L\left(\beta \alpha_{0}, 1\right)}{\sum_{\beta \in \mathbf{F}_{r}^{*}} \beta L\left(\beta \alpha_{1}, 1\right)} .
$$

But, in ([7]), it was shown that $e\left(\lambda \alpha_{1}\right) / e\left(\lambda \alpha_{0}\right)$ is an invertible function (unit!) on $M_{\mathfrak{P}^{n}}^{1}$. Due to the work of Galovich-Rosen, Hayes and the author, it is now known that these units generate groups of finite index. There are further deep connections with Stickelberger elements, (see [8]).

4. The relative zeta-functions. In this chapter we introduce the study of "relative" zeta-functions. These functions play the role classically played by the Dedekind zeta-function of a number field. In particular, once the basic questions of analyticity and rationality are settled for these functions, it appears almost certain that their special values will fit into an Iwasawa set-up completely similar to that envisioned for totally-real number fields.

4.1. Definition. Let $[L: k]<\infty$ and let $\theta$ be the ring of $A$-integers in $L$. Let $\mathbf{N}$ be the norm map on ideals. We set

$$
\zeta_{\mathfrak{O}}(s)=\sum_{\substack{\mathfrak{B} \subset \mathcal{O} \\ \mathfrak{B} \text { an ideal }}}(\mathbf{N} \mathfrak{B})^{-s}=\prod_{\mathfrak{B} \text { prime }}\left(1-(\mathbf{N} \mathfrak{B})^{-s}\right)^{-1} .
$$

We call $\zeta_{\vartheta}$, the zeta-function of $\Theta$ over $A$. We conjecture it may always be extended to an entire function.

If $L$ is abelian over $k$, we can, in certain cases, show this conjecture by reduction to $L$-series in a procedure similar to the classical one. We will not prove the most general case here, but will content ourselves with the illustrative case of the schemes $M_{\mathfrak{P}^{n}}^{1}$ of ([2]), (" $n$ " here is distinct from " $r=p^{n}$ "). Recall that $M_{\mathfrak{P}^{n}}^{1}$ is Galois, abelian over $k$. There is a map $M_{\mathfrak{R}^{n}}^{1} \rightarrow M_{1}$ which is totally ramified at all primes above $\mathfrak{P}$ and is étale elsewhere. The Galois group of this map is isomorphic to $\left(A / \mathfrak{B}^{n}\right)^{*} / \mathbf{F}_{r}^{*}$. The Galois group, $G$, of $M_{\mathfrak{B}^{n}}^{1} / A$ is, in the notation of 2.3 , $\cong$ $\left(I(\mathfrak{B}) / P\left(\mathfrak{B}^{n}\right)\right) / \mathbf{F}_{r}^{*}$, in accordance with class-field theory. Finally $M_{\mathfrak{P}^{n}}^{1}$ 
splits totally at $\infty$. We have an exact sequence

$$
0 \rightarrow \mathbf{F}_{r}^{*} \rightarrow\left(A / \mathfrak{S}^{n}\right)^{*} \rightarrow G \rightarrow \operatorname{Gal}\left(M_{1} / A\right) \rightarrow 0 ;
$$

with $\operatorname{Gal}\left(M_{1} / A\right) \cong\{$ class group of $A\}$. Let $\alpha=D(\mathfrak{B}), U_{1}(n)=\{x \in$ $\left.A_{\mathfrak{P}}^{*} \mid x \equiv 1\left(\mathfrak{B}^{n}\right)\right\}, U_{1}=1$-units in $A_{\mathfrak{P}}^{*}$, and $\mu_{r^{\alpha}-1}=\left(r^{\alpha}-1\right)$ st roots of 1 . Then,

$$
\left(A / \mathfrak{B}^{n}\right)^{*} \cong \mu_{r^{\alpha}-1} \times U_{1} / U_{1}(n),
$$

with $U_{1} / U_{1}(n)$ a finite $p$-group.

It is clear that any $\bar{k}$-valued multiplicative character of $G /\left(U_{1} / U_{1}(n)\right)$ extends uniquely to $G$ since $\operatorname{ch}(\bar{k})=p$. (The reader should not confuse these characters with those used to define $\mathfrak{U}^{-s}$.) Thus a character $\chi$ on $G$ arises from a primitive character $\chi_{0}$ on either $\operatorname{Gal}\left(M_{\mathfrak{B}}^{1} / A\right)$ or $\operatorname{Gal}\left(M_{1} / A\right)$. If it arises from $M_{\mathfrak{P}}^{1}$ and not $M_{1}$, we say $\mathfrak{B}$ is its level, otherwise, we say $\mathfrak{B}^{0}$ is its level.

Now $\#\left(\operatorname{Gal}\left(M_{\mathfrak{B}^{n}}^{1} / A\right)\right)=\left(\left(r^{n D(\Re)}-1\right) /(r-1)\right) h$, with $h=$ class number of $A$. Let $\# G=g_{0} \cdot g_{1}$, with $g_{0}$ a power of $p$ and $\left(p, g_{1}\right)=1$. Let $h=h_{0} h_{1}$ with the same restrictions.

For convenience, let $\zeta_{M_{\mathfrak{x}^{n}}^{1}}(s)$ denote the zeta-function of $\Gamma\left(M_{\mathfrak{B}^{n}}^{1}, \mathcal{O}_{M_{\mathfrak{B} n}^{1}}\right) / A$.

4.2. THEOREM. $\zeta_{M_{\mathfrak{B} n}^{1}}(A)=\left(\Pi_{\chi} L\left(\chi_{0}, s\right)^{g_{0}}\right)\left(1-\mathfrak{P}^{-f s}\right)^{\alpha}, \quad \alpha=$ $j\left(g_{0} / h_{0}-1\right)$; where $f=\{$ ord $\mathfrak{B}$ in the ideal class group of $A\}$ and $f \cdot j=h$.

Proof. First, let $\mathfrak{B}_{1}$ be a prime $\neq \mathfrak{B}$ of $A$. The primes above $\mathfrak{B}_{1}$ in $M_{\mathfrak{P}^{n}}^{1}$ are unramified. Thus, in $\Gamma\left(M_{\mathfrak{P}^{n}}^{1}, \mathcal{O}_{M_{\mathfrak{k} n}^{1}}\right)$,

$$
\left(\mathfrak{B}_{1}\right)=\left(\mathfrak{B}_{1} \cdots \mathfrak{B}_{u}\right),
$$

with $\mathfrak{B}_{1}, \ldots, \mathfrak{B}_{u}$ prime and $\mathbf{N}_{\mathfrak{B}_{\mathfrak{u}}}=\cdots=\mathbf{N}_{\mathfrak{B}_{u}}=\mathfrak{B}_{1}^{f_{1}}$, and $f_{1} \cdot r=\# G$. The contribution to $\zeta_{M_{\mathfrak{B} n}^{1}}(s)$ is therefore

$$
\left(1-\left(\mathfrak{P}_{1}^{f_{1}}\right)^{-s}\right)^{u}
$$

Let $t=\mathfrak{B}_{1}^{-s}$; so the contribution is

$$
\left(1-t^{f_{1}}\right)^{-u}
$$

On the other hand, $\mathfrak{B}_{1}$ generates a subgroup $H$ of order $f_{1}$ in $G$. Let $f_{1}=f_{2} f_{3}$ with $f_{2} \mid g_{0}$ and $f_{3} \mid g_{1}$. As $\mathfrak{P} \neq \mathfrak{P}_{1}$, the values of a character $\chi$ on $\mathfrak{B}_{1}$ is the same as that of the induced primitive character. There are $f_{3}$ characters on $H$, each extending in $g_{1} / f_{3}$ ways.

Now

$$
\left(1-t^{f_{3}}\right)=\prod_{\zeta^{f_{3}}=1}(1-\zeta t)
$$


Thus,

$$
\left(1-t^{f_{3}}\right)^{g_{1} / f_{3}}=\prod_{\chi}\left(1-\chi_{0}(\mathfrak{B}) t\right) .
$$

On the other hand, we see

$$
\left(\left(1-t^{f_{3}}\right)^{g_{1} / f_{3}}\right)^{g_{0}}=\left(1-t^{f_{3}}\right)^{\alpha}, \quad \alpha=g_{1} g_{0} / f_{3} .
$$

As the characteristic is $p$, this in turn equals

$$
\left(1-t^{f_{3} f_{2}}\right)^{\alpha / f_{2}} \text {. }
$$

But, $f_{3} f_{2}=f_{1}$ and $\alpha / f_{2}=u$. This completes the result here.

Next let $\mathfrak{P}_{1}=\mathfrak{B}$. Then, in $\Gamma\left(M_{\mathfrak{P}^{n}}^{1}, \Theta_{M_{\mathfrak{1}}^{1}}\right)$,

$$
(\mathfrak{B})=\left(\mathfrak{B}_{1} \cdots \mathfrak{B}_{j}\right)^{e},
$$

with $\mathbf{N}\left(\mathfrak{B}_{i}\right)=\mathfrak{B}^{f}$ all $i, f \cdot j=h$ and $f \cdot j \cdot e=\# G$. Indeed, over $M_{1}$, there is total ramification. The contribution above $\mathfrak{B}$ is therefore

$$
\left(1-\mathfrak{P}^{-f s}\right)^{-j} \text {. }
$$

Set $t=\mathfrak{B}^{-s}$ and let $; f=f_{0} f_{1}, j=j_{0} j_{1}$ with the usual restrictions. As before,

$$
\left(1-t^{f_{1}}\right)^{j_{1}}=\prod_{\chi}(1-\chi(\mathfrak{B}) t)
$$

with $\chi$ running through the characters of $\operatorname{Gal}\left(M_{1} / A\right)$. Thus, as $j_{1}=h_{1} / f_{1}$,

$$
\begin{aligned}
\prod_{\chi}(1-\chi(\mathfrak{P}) t)^{g_{0}} & =\left(\left(1-t^{f_{1}}\right)^{h_{1} / f_{1}}\right)^{\delta_{0}}=\left(1-t^{f_{1}}\right)^{h_{1} g_{0} / f_{1}} \\
& =\left(1-t^{f}\right)^{\frac{h \frac{g_{0}}{h_{0}}}{f}}=\left(1-t^{f}\right)^{j \frac{g_{0}}{h_{0}}} .
\end{aligned}
$$

The result now follows directly.

\subsection{Corollary. $\zeta_{M_{\mathfrak{p}^{n}}^{1}}(s)$ is entire.}

4.4. Remark. Obviously, for non-abelian extensions of $k$ the above procedure fails. It is not unreasonable to expect, however, that the Riemann-Roch theorem, together with results like 1.2 , will eventually establish the results in general.

5. The theory when $s_{1}$ is a negative integer. Our goal here is to establish the basic algebraicity of our function at negative integers. The reader will see that at the negative integers our functions possess rational 
"polynomials" as opposed to just "values". These polynomials are then interpolated at all primes of $k$.

5.1. The algebraicity of L-series for $s_{1}=-i$. Let $\alpha \in A, a, b \in \mathbf{N}$, $H \subseteq A$ an ideal, and $S(\alpha, H, a, b)=\Sigma_{n \equiv \alpha(H) ; D(n)=b} n^{a}$. As a simple corollary of 1.2 and the Riemann-Roch theorem, we see that for $b$ sufficiently large $S(\alpha, H, a, b)=0$.

If we multiply $S(\alpha, H, a, b)$ by $\pi_{1}^{b}$, we have the same sum over 1 -units. Thus the following result is immediate.

5.1.1. THEOREM. Let $L(s)$ be one of the functions of 2.3.1 or 2.3.2. Then $L\left(s_{0},-i\right)$ is a Laurent-polynomial for $i \in \mathbf{N}$.

Now let $\mathfrak{A}$ be a fractional ideal, $n_{1}, n_{2} \in \mathfrak{A}$ and $I \subseteq A$ an ideal. Let $n_{1}, n_{2} \in \mathfrak{A}$ with $D\left(n_{1}\right)=D\left(n_{2}\right)=j, n_{1} \equiv n_{2}(I \mathscr{U})$ and $\left\langle n_{1}\right\rangle \equiv\left\langle n_{2}\right\rangle\left(\pi^{m}\right)$. Then $\lambda=n_{1}-n_{2} \in I \mathfrak{A}$ and $\pi^{j} \lambda \in\left(\pi^{m}\right)$, or $D(\lambda) \leq j-m$. Conversely, given $\lambda$, we can add it to $n_{1}$ to obtain an $n_{2}$ with the above property. The set of such $\lambda$ is an $\mathbf{F}_{r}$-vector space and if we use 1.2 on each coset, along with the binomial theorem, we conclude

5.1.2. THEOREM. Let $L(s)$ be one of the functions of 2.3.1 or 2.3.2. Then $L\left(s_{0},-i\right)$ is a Laurent-polynomial for $i \in \mathbf{N}$.

5.1.3. COROLlARY. $\zeta_{M_{\mathfrak{*}}^{1} n}\left(s_{0},-i\right)$ is a Laurent-polynomial for $i \in \mathbf{N}$.

Proof. This is immediate from 5.1.1 and 4.2.

As a corollary of the above, we have the following method of calculation: Let, for instance, $L(s)=\zeta(s)$ and let

$$
X=\left\{\mathfrak{A}_{0}, \ldots, \mathfrak{A}_{m}\right\} .
$$

Then, we have

$$
\zeta\left(\mathfrak{U}_{j},-i\right)=\sum_{v>-\infty} \sum_{\substack{h \in \hat{\mathfrak{A}}_{j} \\ D(h)=v}} h^{i},
$$

is a finite sum and it belongs to $k$.

5.2. Some trivial zeros for zeta and L-functions. We begin with the zeta-function. Fix a collection $X=\left\{\mathfrak{A}_{0}, \ldots, \mathfrak{A}_{m}\right\}$. Then we have

$$
\zeta(s)=\sum_{i=0}^{m} \zeta(\mathfrak{A}, s) \mathfrak{A}_{\imath}^{-s} .
$$


Set

$$
Z\left(s_{0},-i\right)=\zeta\left(\pi^{i} s_{0},-i\right)
$$

Thus,

$$
\begin{aligned}
Z\left(s_{0},-i\right) & =\sum_{c=0}^{m}\left(\sum_{j>-\infty} s_{0}^{-j} \sum_{\substack{n \in \hat{A}_{c} \\
D(n)=j}} n^{i}\right) \mathfrak{U}_{c}^{l} \\
& =\sum_{c=0}^{m} Z\left(\mathfrak{A}_{c},\left(s_{0},-i\right)\right) \mathfrak{A}_{c}^{i}
\end{aligned}
$$

In particular, the sum in parentheses is a Laurent-polynomial.

Our first result is a non-vanishing result:

5.2.1. Lemma. $Z\left(\mathfrak{A}_{c},(1,0)\right)=\zeta\left(\mathfrak{U}_{c}, 0\right)=1$ for all $c$.

Proof. There always exists a unique monic of least degree in $\hat{\mathfrak{A}}_{c}$. The result is obvious upon taking multiplicities into account.

Let $H \subseteq A$ be an ideal. We let $S(0, H,(r-1), j)$ be defined as in 5.1, with $j$ so large that the sum is 0 .

5.2.2. LEMMA.

$$
S(0, H,(r-1), j)=-\sum_{\substack{(n)<j \\ n \in H}} n^{(r-1)} .
$$

Proof. Let $f$ be a fixed element of degree $N(r-1)$ in $H$. Any other may be written as $f+\alpha h$, with $h \in H$ of smaller degree and $\alpha \in \mathbf{F}_{r}$. Then

$$
(f+\alpha h)^{(r-1)}=\sum_{i=0}^{(r-1)} f^{i}(\alpha h)^{(r-1)-i}\left(\begin{array}{c}
r-1 \\
i
\end{array}\right) .
$$

Summing over $\alpha$ kills the terms corresponding to $i>0$. We are left with $-h^{(r-1)}$. Summing over $h$ finishes the proof.

5.2.3. CoRollary. $\zeta\left(\mathfrak{A}_{c},-\left(r^{c}-1\right)\right)=Z\left(\mathfrak{A}_{c},(1,-(r-1))\right)=0$.

Proof. Upon multiplying by a constant, we reduce to having $\mathfrak{A}_{c}=H$ be integral. As

$$
S(0, H,(r-1), j)=0,
$$

we see the result follows from 5.2.2.

5.2.4. TheOREM. $\zeta(-i)=0$ unconditionally for $i \equiv 0(r-1), i>0$. 
Proof. Of course, we need only check the result for the corresponding components. But, by induction, this follows as in 5.2.2, 5.2.3.

Thus, in this case, we obtain relations between the zeros of the different components.

We turn now to the case of $L$-series associated to characters $\chi$. For convenience, we work only with characters on

$$
q(\mathfrak{B}) / P\left(\mathfrak{B}^{m}\right)
$$

$\mathfrak{B}$ prime. However, our arguments easily generalize to the case where $\mathfrak{P}$ is replaced by an arbitrary $I$.

Let $X=\left\{\mathfrak{A}_{0}, \ldots, \mathfrak{A}_{m}\right\},\left(\mathfrak{A}_{i}, \mathfrak{B}\right)=1$ all $i$. Suppose that for $\zeta \in \mathbf{F}_{r}^{*}$, $\chi(\zeta)=\zeta^{l}$, where we think of $\mathbf{F}_{r}^{*} \subseteq \mathscr{G}(\mathfrak{B}) / P\left(\mathfrak{P}^{m}\right)$ in the obvious fashion.

5.2.5. THEOREM. If $0 \leq l<r-1$, then $L(\chi,-i)=0$ unconditionally, for $i \equiv-l(r-1)$.

Proof. As before, we may restrict ourselves to handling only the components of $L$. These are of the form

$$
\sum_{j>-\infty}\left(\sum_{\substack{n \in \hat{\mathscr{A}}_{c} \\ D(n)=j}} \chi(n) n^{i}\right),
$$

where $\chi$ is extended to $\hat{\mathfrak{U}}{ }_{c}$ in the obvious fashion.

Assume first that each residue class of $\hat{\mathfrak{U}}_{c} \bmod \mathfrak{P}^{m} \hat{\mathfrak{U}}_{c}$ has representative of degree $<f, f=\min _{0 \neq \beta \in \mathfrak{P}^{m} \hat{\mathfrak{Q}}_{c}}\{D(\beta)\}$. Our sum becomes

$$
\begin{aligned}
& \sum_{\alpha \in \mathfrak{A}_{c} / \mathfrak{P}^{m} \mathfrak{A}_{c}} \chi(\alpha)\left(\sum_{j \geq-\infty} \sum_{\substack{n \in \hat{\mathfrak{A}}_{c} \\
n \equiv \alpha\left(\mathfrak{B}^{m} \hat{\mathfrak{A}}_{c}\right) \\
D(n)=j}} n^{i}\right) \\
& =\sum_{\alpha} \chi(\alpha)\left(\sum_{j>-\infty} \sum_{\substack{D(h)=j \\
h \in \mathfrak{P}^{m} \hat{\mathfrak{A}}_{c}}}(\alpha+h)^{i}\right)+\sum_{\substack{\alpha \in \hat{\mathfrak{A}}_{c} / \mathfrak{P}^{m} \hat{\mathfrak{A}}_{c} \\
\alpha \text { monic }}} \chi(\alpha) \alpha^{i} \\
& =\sum_{\alpha} \chi(\alpha)\left(\sum_{j>-\infty} \sum_{\substack{D(h)=j \\
h \in \mathfrak{P}^{m} \hat{\mathfrak{U}}_{c}}} \sum_{t=0}^{i} \alpha^{t} h^{i-t}\left(\begin{array}{l}
i \\
t
\end{array}\right)\right)+\sum_{\alpha \text { monic }} \chi(\alpha) \alpha^{i} \\
& =\sum_{\alpha} \chi(\alpha)\left(\sum_{t=0}^{i} \alpha^{t}\left(\begin{array}{l}
i \\
t
\end{array}\right) \zeta\left(\hat{\mathfrak{B}}^{m} \mathfrak{A}_{c},-(i-t)\right)\right)+\sum_{\alpha \text { monic }} \chi(\alpha) \alpha^{i} .
\end{aligned}
$$


Let $\left(\hat{\mathfrak{U}}_{c} / \mathfrak{P}^{m} \hat{\mathfrak{U}}_{c}\right)^{*}$ be defined as

$$
\hat{\mathfrak{A}}_{c} / \mathfrak{P}^{m} \hat{\mathfrak{U}}_{c}-\mathfrak{B} \hat{\mathfrak{U}} \hat{c}_{c} / \mathfrak{B}^{m} \hat{\mathfrak{U}}_{c} .
$$

Let $\left\{\alpha_{1}, \ldots, \alpha_{v}\right\}$ be monic representatives of

$$
\mathbf{F}_{r}^{*} \backslash\left(\hat{\mathfrak{A}}_{c} / \mathfrak{P}^{m} \hat{\mathfrak{U}}_{c}\right)^{*} .
$$

Our sum becomes,

$$
\sum_{j} \chi\left(\alpha_{j}\right)\left(\sum_{\zeta \in \mathbf{F}_{r}^{*}} \zeta^{l} \sum_{t=0}^{i} \zeta^{t} \alpha_{j}^{t}\left(\begin{array}{l}
i \\
t
\end{array}\right) \zeta\left(\hat{\mathfrak{P}}^{m} \mathfrak{A}_{c},-(i-t)\right)\right)+\sum_{\alpha \text { monic }} \chi(\alpha) \alpha^{i}
$$

Now if $(r-1) \mid(i-t),(i-t)>0$, then, by 5.3.3,

$$
\zeta\left(\hat{\mathfrak{B}}^{m} \mathfrak{U}_{c},-(i-t)\right)=0 .
$$

If $(r-1)+(i-t) \Rightarrow(r-1)+(l+t) \Rightarrow \Sigma \xi^{l+t}=0$. If we note that $\zeta\left(\hat{\mathfrak{P}}^{m} \mathfrak{U}_{c}, 0\right)=1$, and that $(r-1) \mid(i+l)$, by assumption, then we are left with

$$
\sum \chi\left(\alpha_{j}\right) \sum \zeta^{(r-1)} \alpha_{J}^{i}+\sum_{\alpha \text { monic }} \chi(\alpha) \alpha^{i}=0
$$

Next, suppose there exist $\alpha$ with $D(\alpha)>f ; \alpha$ smallest in its class. Our sum becomes

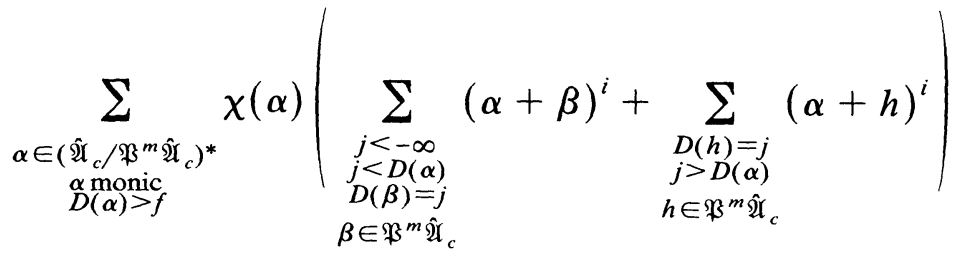

$$
\begin{aligned}
& +\sum_{\substack{\alpha \in\left(\hat{\mathfrak{U}}_{c} / \mathfrak{B}^{m} \hat{\mathfrak{U}}_{c}\right)^{*} \\
\text { non-monic } \\
D(\alpha)>f}} \chi(\alpha)\left(\sum_{\substack{D(h)=j \\
j>D(\alpha) \\
h \in \mathfrak{\beta}^{m} \hat{\mathfrak{A}}_{c}}}(\alpha+h)^{i}\right) \\
& +\sum_{\substack{\alpha \in\left(\hat{\mathfrak{U}}_{,} / \mathfrak{P}^{m} \hat{\mathfrak{U}}_{c}\right)^{*} \\
D(\alpha)<f}} \chi(\alpha)\left(\sum_{\substack{j>-\infty \\
h \in \mathfrak{\beta}^{m} \hat{\mathfrak{A}}_{c} \\
D(h)=j}}(\alpha+h)^{i}\right)+\sum_{\alpha \text { monic }} \chi(\alpha) \alpha^{i} .
\end{aligned}
$$


Now suppose $\alpha$ monic $D(\alpha)>f$; obviously if $1 \neq \zeta \in \mathbf{F}_{r}^{*}$, then $\zeta \alpha$ is of the same degree but non-monic. Our sum with respect to these $\alpha$ becomes

$$
\begin{aligned}
& \chi(\alpha) \sum_{\substack{j<D(d) \\
D(h)=j \\
\alpha \text { monic }}}(\alpha+h)^{i}+\chi(\alpha) \sum_{\substack{j>D(d) \\
h \in \mathfrak{P}^{m}}}(\alpha+h)^{i} \\
& +\sum_{\zeta \in F_{r}^{*}-1} \chi(\alpha) \zeta^{-i} \sum_{\substack{j<D(\alpha) \\
D(h)=j}}(\zeta \alpha+h)^{i}+(\text { rest }) .
\end{aligned}
$$

But, $\alpha^{-i}=\zeta^{l}$, so $\chi(\alpha) \zeta^{l}=\chi(\zeta \alpha)$. Thus, we can express our sum as we did above and proceed in the same fashion to finish the proof.

\subsection{Some additional results with $A=\mathbf{F}_{r}[T]$}

When $A=\mathbf{F}_{r}[T]$, we can say much more.

\subsubsection{LEMMA.}

$$
Z\left(s_{0},-i\right)=1-\sum_{\substack{b=0 \\
(r-1) \mid(i-b)}}^{i-1}\left(\begin{array}{l}
i \\
b
\end{array}\right) T^{b} s_{0}^{-1} Z\left(s_{0},-b\right),
$$

for $i \in \mathbf{N}^{+}$.

Proof. Write $n=T h+\zeta$ with $D(h)+1=D(n), \zeta \in \mathbf{F}_{r}$, and expand out the additive formula for $Z\left(s_{0},-i\right)$. Upon noticing

$$
\sum \zeta^{j}=\left\{\begin{array}{c}
0 \text { if }(r-1) \nmid j \\
-1 \text { otherwise }
\end{array}\right\}, \quad \text { the result follows. }
$$

If we put $s_{0}=1$, we obtain a formula for $\zeta(-i)$. If we apply $d / d s_{0}$, we obtain a formula for $\left(d / d s_{0}\right) Z\left(s_{0},-i\right)$.

5.3.2. Definition. We set $\beta(0), \beta(1)=1$ and for $i>1$

$$
\beta(i)=1-\sum_{\substack{b=0 \\
(r-1) \mid(i-b)}}^{i-1}\left(\begin{array}{l}
i \\
b
\end{array}\right) T^{b} \beta(b) .
$$

5.3.3. LeMMA. Let $i \in \mathrm{N}^{+}$. Then

$$
\beta(i)=\left\{\begin{array}{l}
\zeta(-i) \quad \text { if }(r-1)+i \\
\frac{d}{d s_{0}} Z(1,-i) \quad \text { if }(r-1) \mid i .
\end{array}\right.
$$


Proof. This follows from the above and 5.2.4.

Clearly $\beta(i)(0)=1$ for $i>0$. Thus, we have for $i \equiv 0(r-1)$,

5.3.4. Proposition. The zero of $Z\left(s_{0},-i\right)$ at $s_{0}=1$ is simple.

This is exactly as for the Riemann zeta-function.

Let now $r=p$.

5.3.5. Definition. (1) Let $j \in \mathbf{N}^{+}$be written as $\sum_{i=0}^{n} c_{l} p^{i}, 0 \leq c_{i}<p$. We set $S(j)=\Sigma c_{i}$.

(2) Let $j$ be written as $\Sigma p^{e_{i}}, e_{1} \leq e_{2} \leq \cdots$, and suppose $S(j)>$ $(p-1)$, with each $e_{i}$ occurring at most $(p-1)$ times. Set

$$
\rho(j)=j-\sum_{i=1}^{p-1} p^{e_{i}}, \quad \rho^{2}=\rho \circ \rho, \text { etc. }
$$

Using formula on congruences of binomial coefficients, Emory Thomas has shown the following:

5.3.6. TheOREM. (1) Let $i \in \mathbf{N}^{+}$with $S(i) \leq p-1$. Then $\beta(i)=1$.

(2) Let $S(i)>p-1$ and let $S(i)=c(p-1)+d$, with $0<c$ and $1 \leq d \leq p-1$. Then $D(\beta(i))=\sum_{e=1}^{c} \rho^{e}(i)$.

The reader should compare the above with 3.2.5.

5.4. The v-adic theory and interpolation. We now discuss the interpolation of our functions at the finite primes. The reader will note the remarkable similarity to the $\pi$-adic theory. In particular, the $v$-adic functions will also turn out to be entire in a sense similar to the $\pi$-adic sense.

Let $\mathfrak{P}$ be a finite prime of degree $\alpha$ and let $v$ be the associated plane.

\subsubsection{Definition. We set}

$$
S_{v}=\mathbf{Z} /\left(r^{\alpha}-1\right) \times \mathbf{Z}_{p}=\lim _{\overleftarrow{i}} \mathbf{Z} /\left(\left(r^{\alpha}-1\right) p^{i}\right) .
$$

We call $S_{v}$ the character-space at $v$. The integer powers are dense in $S_{v}$.

5.4.2. Proposition. Let $i_{1}, i_{2} \in \mathbf{N}$ with $i_{1}-i_{2} \in\left(r^{\alpha}-1\right) p^{e}$. Let $n \in k$ be a v-adic unit. Then

$$
n^{i_{1}}-n^{l_{2}} \in \mathfrak{P}^{p^{e}}
$$


5.4.3. Corollary. The function $i \rightarrow n^{i}$ interpolates to a continuous function $S_{v} \rightarrow A_{v}^{*}$.

Note the extremely strong congruences given by 5.4.2.

$S_{v}$ is the exact $v$-adic analogue of $S_{\infty}$ and of the classical $p$-adic character space. What is surprising about the present situation is that our functions naturally occur on a much larger two-variable space.

\subsubsection{Definition. We set $X_{v}=k_{v}^{*} \times S_{v}$.}

Let $C$ be a fixed integral ideal and let $\mathfrak{A}$ be prime to $\mathfrak{B}$. We now define $\mathfrak{U}^{x_{1}}$, the $v$-adic version of $\mathfrak{U}^{s_{1}}$. Let

$$
\hat{g}\left(C \pi^{m}\right)_{v}=\underset{\leftarrow}{\lim } \mathscr{}(C \mathfrak{P}) / P\left(\pi^{m} C \mathfrak{P}^{j}\right)
$$

We have an exact sequence,

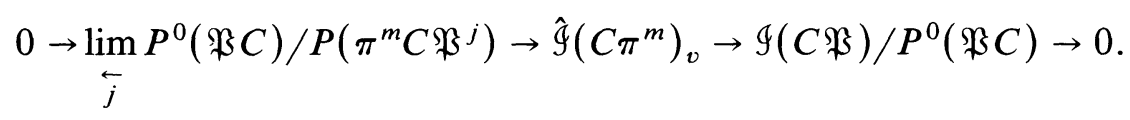

But,

$$
\underset{\leftarrow}{\lim } P^{0}(\mathfrak{B} C) / P\left(\mathfrak{P}^{j} C \pi^{m}\right) \cong U=\text { units in } k_{v} .
$$

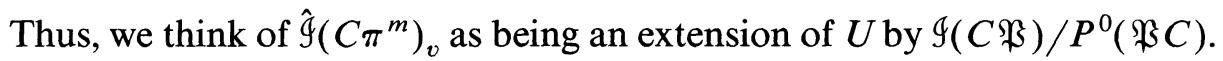
As in the $\pi$-adic case, for simplicity of exposition, we now set $C=A$, $m=0$ and $\hat{g}_{v}=\hat{g}\left(C \pi^{m}\right)_{v}$.

We now consider only those continuous $\bar{k}_{v}$-valued characters (or, more generally, maps to $\left.\operatorname{GL}\left(n, \bar{k}_{v}\right)\right)$ that induce $n^{x_{1}}$ on the units, etc.

Let $X=\left\{\mathfrak{A}_{0}, \ldots, \mathfrak{A}_{m}\right\},\left(\mathfrak{U}_{i}, \mathfrak{P}\right)=1$ all $i$, be a collection of ideal class representatives.

5.4.5. Definition. Let $x=\left(x_{0}, x_{1}\right) \in X_{v}$.

(1) We set

$$
\zeta_{v}(x)=\prod_{\substack{\mathfrak{Q} \neq \mathfrak{P} \\ \mathfrak{B} \text { prime }}}\left(1-x_{0}^{-D(\mathfrak{B})} \mathfrak{B}^{-x_{1}}\right)^{-1}=\sum_{\substack{(\mathfrak{A}, \mathfrak{P})=1 \\ \mathfrak{A} \subseteq A}} x_{0}^{-D(\mathfrak{A})} \mathfrak{U}^{-x_{1}}
$$

$\zeta_{v}(x)$ converges for all $x$ with $v\left(x_{0}\right)>0,(v$ considered additively).

(2) Let $D$ be an ideal divisible by $\mathfrak{B}$.

(a) Let $\delta \in G(D) / P\left(\pi^{m} D\right)$. We set

$$
\zeta_{v}(\delta, x)=\sum_{\substack{\mathfrak{A} \in \delta \\ \mathfrak{U} \subseteq A}} x_{0}^{-D(\mathfrak{A})} \mathfrak{U}^{-x_{1}}
$$


(b) Let $\delta_{1} \in \mathscr{G}(D) / P^{0}(D)$. We set

$$
\zeta\left(\delta_{1}, x\right)=\sum_{\delta \rightarrow \delta_{1}} \zeta(\delta, x) .
$$

(c) Let $f$ be a $\vec{k}$-valued function on $9(D) / P\left(D \pi^{m}\right)$. We set

$$
L_{v}(f, x)=\sum_{\substack{\mathfrak{A}, D)=1 \\ \mathfrak{U} \subseteq A}} f(\mathfrak{U}) x_{0}^{-D(\mathfrak{U})} \mathfrak{U}^{-x_{1}}
$$

Note that $L_{v}(f, x)$ can easily be expressed in terms of partial zeta-functions. All functions clearly converge for $v\left(x_{0}\right)>0$.

It is clear, for instance, that

$$
\zeta_{v}(x)=\sum_{i=0}^{m} \zeta_{v}\left(\mathfrak{U}_{i}, x\right)\left(x_{0}^{-D\left(\mathfrak{A}_{i}\right)} \mathfrak{A}_{\imath}^{-x_{1}}\right)
$$

where

$$
\zeta_{v}\left(\mathfrak{A}_{1}, x\right)=\sum_{\substack{n \in \hat{A}_{l} \\(n, \mathfrak{P})^{\prime}=1}} x_{0}^{-D(n)} n^{-x_{1}}=\sum_{j>-\infty} x_{0}^{-J}\left(\sum_{\substack{n \in \hat{A}_{l} \\(n, \mathfrak{P})=1 \\ D(n)=j}} n^{-x_{1}}\right)
$$

From now on, we use the symbol $\Sigma^{\prime}$ to mean that we sum over the elements prime to $\mathfrak{B}$. We always sum by using cosets mod $\mathfrak{B}$ with generators prime to $\mathfrak{B}$. So, we can use previous vanishing results to conclude that if $L(x)$ is one of the above functions, then for $x_{1}=-i$, $i \in \mathbf{N}, L(x)$ is a Laurent polynomial.

Before discussing analyticity, we want to make the connection with the $\pi$-adic functions explicit. We need only do so in the illustrative case of the zeta-function: Let $x=\left(x_{0}, x_{1}\right)$ with $x_{1}=-i, i \in \mathbf{N}$, and $x_{0} \in k^{*}$. Let $s=\left(\pi^{i} s_{0}, s_{1}\right), s_{0}=x_{0}$ and $s_{1}=x_{1}=-i$. Then, we have an equality of Laurent-polynomials.

$$
\text { 5.4.6. } \begin{aligned}
\zeta_{v}(x) & =\left(1-\mathfrak{P}^{-s}\right) \zeta(s)=\left(\sum_{c=0}^{m} \zeta\left(\mathfrak{U}_{c}, s\right) \mathfrak{A}_{c}^{-s}\right)\left(1-\mathfrak{P}^{-s}\right) \\
& =\sum_{c=0}^{m}\left(\sum_{\jmath>-\infty} s_{0}^{-J}\left(\sum_{\substack{n \in \hat{\mathfrak{A}}_{c} \\
D(n)=j}}^{\prime} n^{l}\right)\right) \mathfrak{U}_{c}^{-s} ;
\end{aligned}
$$


where we have equated $\pi^{-D\left(\mathfrak{H}_{c}\right)}\left\langle\mathfrak{U}_{c}\right\rangle^{l}$ with $\mathfrak{U}_{c}^{i}$; one symbol considered $\pi$-adically and one $v$-adically. (We leave it to the reader to make this a rigorous statement concerning characters.) For instance, when $A=\mathbf{F}_{r}[T]$, $\zeta_{v}(x)=\left(1-\mathfrak{P}^{-s}\right) Z\left(s_{0},-i\right)$.

Therefore, we see that for $x_{1}=-i, i \in \mathbf{N}$, our $v$-adic functions are, up to a possible Euler factor, special values of our $\pi$-adic functions for $x_{0} \in k$.

5.4.7. THEOREM. The functions of 5.4.5 interpolate to entire functions on $X_{v}$.

Proof. As in the $\pi$-adic case, we reduce to handling sums of the following form: Let $\mathfrak{A} \subseteq k$ be prime to $\mathfrak{P}$ and let $\alpha \in \mathfrak{A}-\mathfrak{P} \mathfrak{A}$. Then, we need only show sums of the form

$$
\sum_{j>-\infty} x_{0}^{-j}\left(\sum_{\substack{D(n)=j \\ n \in \mathfrak{A} \\ n \equiv \alpha(\mathfrak{B} \mathscr{A}) \\\langle n\rangle \equiv 1\left(\pi^{n}\right)}} n^{-x_{1}}\right)
$$

converge for all $x_{0} \neq 0$. We can, as before, consider $\mathscr{U}$ as being integral.

Now let $n=\omega(n)\langle n\rangle_{v}$, with $\langle n\rangle_{v}$ a 1-unit, and $\omega(n)=$ the Teichmüller representative. Then,

$$
n^{-x_{1}}=\omega(n)^{\alpha_{0}}\langle n\rangle_{v}^{\alpha_{1}}
$$

with

$$
\alpha_{0} \in \mathbf{Z} /\left(r^{\alpha}-1\right) \text { and } \alpha_{1} \in \mathbf{Z}_{p} .
$$

As $n \equiv \alpha(\mathfrak{P} \mathfrak{U})$, we have $\omega(n)=\omega(\alpha)$. Further, if $D(n)>D(\alpha)$, then

$$
n=\alpha+h,
$$

with $h \in \mathfrak{P} \mathfrak{A}$. Also, if $j=D\left(n_{1}\right)=D\left(n_{2}\right)$, and $n_{1} \equiv \alpha \equiv n_{2}$, then

$$
\pi^{j}\left(n_{1}-n_{2}\right) \in\left(\pi^{m}\right) \text {. }
$$

If we let $\langle n\rangle_{v}=1+\left(w_{n}\right)_{v}$, then the coefficient of $x_{0}^{-J}$ becomes

$$
\omega(\alpha)^{\alpha_{0}} \sum_{\substack{D(n)=j \\
n \equiv \alpha(\mathfrak{B} \mathfrak{Q})}}\left(1+\left(w_{n}\right)_{v}\right)^{\alpha_{1}}=\omega(\alpha)^{\alpha_{0}} \sum_{\substack{D(n)=j \\
n \equiv \alpha(\mathfrak{B} \mathfrak{U}) \\
\langle n\rangle \gg 1\left(\pi^{m}\right)}} \sum_{t=0}^{\infty}\left(w_{n}\right)_{v}^{t}\left(\begin{array}{c}
\alpha_{1} \\
t
\end{array}\right) .
$$

The set $\left\{\left(w_{n}\right)_{v}\right\}$ is not an $\mathbf{F}_{r}$-vector space. But, as before, it is a principal homogeneous space over one. Also as before, we can now use the Riemann-Roch theorem and 1.2., $v$-adically, to finish the proof.

Thus, we've obtained the $v$-adic interpolation of the polynomials of the type given in 5.4.6. 
5.4.8. Remarks. (1) By using the Teichmüller representatives, and congruences at $\infty$, it is clear now how to extend our theory to the most general situation where $\infty$ is not necessarily rational. It would be interesting to have such results to compare with.

(2) The same remarks that we made at $\infty$ about values also are valid in the $v$-adic theory. Thus, the $v$-adic valuation of the components is independent of the ideal class representative prime to $\mathfrak{B}$.

The zeroes are also invariant. And, as $\pi$-adically, the components of our entire functions have infinite products over their zero sets, counted with multiplicity.

(3) We stress that each time $x_{0}$ is chosen, we obtain a continuous function from $S_{v} \rightarrow k_{v}$; each function is similar to the classical $p$-adic $L$-series. In particular, if $x_{0}=1$, we obtain the interpolation of our functions at negative integral powers.

Next we discuss some corollaries. Let $\mathfrak{A}$ be a fractional ideal and let $\alpha_{0}, \alpha_{1} \in \mathfrak{B}^{-m} \mathfrak{U}-\mathfrak{B}^{-(m-1)} \mathfrak{A}$. Then in 3.3 .3 , we showed that $\pi$-adic series of the form

$$
\sum_{\beta \in \mathfrak{A}}\left(\alpha_{0}+\beta\right)^{-1} / \sum_{\beta \in \mathfrak{A}}\left(\alpha_{1}+\beta\right)^{-1}
$$

are units in the schemes $M_{\mathfrak{B}^{m}}^{1}$. But we've just shown that if we sum these series by grouping according to degree that they also converge $\mathfrak{P}$-adically. The obvious question is, do these series also converge $v$-adically to the same unit?

Finally we return to the relative zeta-functions of $\S 4$. Theorem 4.2 tells us that, upon removing the $\mathfrak{B}$-factor,

$$
\left(\zeta_{M_{\mathfrak{*}}^{1}{ }^{n}}\right)_{v}(s)=\left(\prod_{\chi} L_{v}\left(\chi^{\prime}, s\right)\right)^{g_{0}}
$$

Thus, as we move up the $\mathfrak{P}^{n}$-tower the $\mathfrak{B}$-adic functions differ by powers of $p$. In particular, they have the same zeroes.

5.5. A remark on functional equations. It is not known whether or not the $\pi$-adic functions satisfy functional equations. However, it seems highly doubtful that if they do satisfy functional equations, that they will be of the classical $s \mapsto 1-s$ form. The point is the following: A classical Dirichlet-series is determined, as a Dirichlet series, by its values at an infinite subset of those positive integers for which it converges. On the other hand, they are not determined by their values at negative integers (e.g., $\zeta_{Q}(2 s)$ ). But, via the functional equation, $\zeta_{Q}(s)$ is determined by its values at the negative integers; and, thus by its interpolation at the finite primes. 
Our zeta-function has a whole polynomial at each negative integer and this polynomial is determined by its rational values that are interpolated at the finite primes. Further, these polynomials also determine by continuity the $\pi$-adic function. Thus, from this point of view, a functional equation along the classical lines is not needed!

On the other hand, there is some rather remarkable evidence linking $\beta(i)$ and $\Gamma(T, i)$, that seems to represent a functional equation of some sort. Let $r=p$, and $1 \leq c \leq p-1$ and $i(c, j)=c p^{j}+\left(p^{j}-1\right)$. Then it is easy to use Thomas's formula to show that for $i=i(c, j), D(\beta(i))=$ $D(\Gamma(T, i))$, and, in fact, there is a fascinating degree of agreement between these functions as the following tables will attest, when $r=3$. We begin with $c=1$ and we let $\overline{\beta(i)}=\beta(i) / \zeta$, where $\zeta$ is the highest coefficient of $\beta(i)$.

\begin{tabular}{r|c|c|c}
\multicolumn{1}{c|}{$i$} & $a=D(\overline{\beta(i)})$ & $b=D(\Gamma(T, i)-\overline{\beta(i)})$ & $a-b$ \\
\hline 1 & 0 & 0 & 0 \\
\hline 5 & 3 & 0 & 3 \\
\hline 17 & 24 & 15 & $3^{2}$ \\
\hline 53 & 123 & 96 & $3^{3}$ \\
\hline 161 & 528 & 447 & $3^{4}$ \\
\hline 485 & 2067 & 1824 & $3^{5}$ \\
\hline. & & &
\end{tabular}

But when $c=2$ we obtain

\begin{tabular}{r|c|c|c}
\multicolumn{1}{c|}{$i$} & $a=D(\overline{\beta(i)})$ & $b=D(\Gamma(T, i)-\overline{\beta(i)})$ & $a-b$ \\
\hline 2 & 0 & 0 & 0 \\
\hline 8 & 6 & 0 & $2 \cdot 3$ \\
\hline 26 & 42 & 24 & $2 \cdot 3^{2}$ \\
\hline 80 & 204 & 150 & $2 \cdot 3^{3}$ \\
\hline$\cdot$ & & &
\end{tabular}

Any explanation for these facts would explain much. It should be noted that it even took a large computer over an hour to grind them out, so a computational explanation is probably ruled out. It is quite remarkable that, unlike the classical situation, we seem to have a deep connection between the values at the negative integers of the zeta-function and the values of the gamma function! (See note at end of paper.) 
6. The connection with distribution theory. The whole of the preceding theory becomes better understood when it is interpreted from the viewpoint of distribution theory. (See 4.4.)

6.1. Distributions and measures. We begin by presenting a very rapid review of distributions and measures. Let $X$ be a compact totally disconnected space, let $L$ be a finite extension of $k_{w}, w$ a place of $k$, and let $\theta \subseteq L$ be the ring of integers.

6.1.1. Definition. An $L$-valued distribution is a finitely additive $L$-valued function on the compact open subsets of $X$. It is a measure iff the set of values on compact opens is bounded.

Let $\mu$ be such a measure and let $f: X \rightarrow L$ be continuous. As $X$ is compact, $f$ is uniformly continuous. It is now simple to see that the Riemann sums associated to $f$ converge. The limit is denoted as $\int_{X} f d \mu$.

If $\mu$ is just a distribution, it is clear we may integrate locally constant functions.

6.1.2. Proposition. Let $X_{1}, X_{2}$ be two compact totally disconnected spaces. Let $\mu$ be a distribution on $X_{1}$ and let $P: X_{1} \rightarrow X_{2}$ be continuous. Then there exists a distribution $P_{*} \mu$ on $X_{2}$ defined by $\int_{X_{2}} f d P_{*} \mu=\int_{X_{1}} f \circ P d \mu$, for any locally constant $f$.

Note that an $\theta$-valued distribution is automatically a measure.

Suppose now that $G=X$ is also a topological abelian group. Then, in the usual way, both the distributions and the measures form an $L$-algebra under convolution. For the moment, call these algebras $D(X)$ and $M(X)$. Let $D_{1}(X)$ be the $\theta$-algebra of $\theta$-valued distributions. So $M(X)=L \otimes$ $D_{1}(X)$.

As $G$ is a group, it has a neighborhood basis of the identity consisting of open subgroups, $\left\{G_{i}\right\}, G_{i} \supseteq G_{i+1}$. Thus, $G / G_{i}$ is finite. It is clear, upon a moment's reflection, that the algebra of distributions on $G / G_{t}$ is precisely the group ring $L\left[G / G_{i}\right]$. If $j>i$, then we obtain a map $L\left[G / G_{j}\right]$ $\rightarrow L\left[G / G_{i}\right]$. We call the inverse limit ring, $L[[G]]$. It is the completed group ring of $G$. The ring $\mathcal{O}[[G]]$ is defined in the obvious fashion.

As measures push forward, the following proposition is obvious.

\subsubsection{Proposition. (a) $\vartheta[[G]] \approx D_{1}(X)$.}

(b) $M(X) \stackrel{\sim}{\rightarrow} L \otimes \vartheta[[G]]$

(c) $D(X) \stackrel{\sim}{\rightarrow} L[[G]]$.

Next let $U_{1} \subseteq L$ be the 1-units and let $R$ be either a field over $\mathbf{F}_{r}$ or a d.v.r. over $\mathbf{F}_{r}$. Then, we form $R\left[\left[U_{1}\right]\right]$ in the obvious fashion. Recall, that, 
$U_{1}$ is an infinite product of copies of $\mathbf{Z}_{p}$. Let $U \subseteq U_{1}$ be an open subgroup, so $G^{\prime}=U / U_{1}$ is a finite $p$-group.

\subsubsection{Lemma. $R\left[G^{\prime}\right]$ is a local ring.}

Proof. Let $G^{\prime}$ be written as a product of prime power subgroups and let $t_{1}, \ldots, t_{j}$ be chosen generators of these subgroups. Then $R\left[G^{\prime}\right]=$ $R\left[t_{1}, \ldots, t_{j}\right] /\left(t_{i}^{p_{i}}-1\right)$. Let $x_{i}=1+t_{i}$. Then

$$
R\left[G^{\prime}\right]=R\left[x_{1}, \ldots, x_{j}\right] /\left(x_{i}^{p^{e_{i}}}=0\right) .
$$

The result is therefore obvious.

6.1.5. Proposition. $R\left[\left[U_{1}\right]\right]$ is a local ring.

Proof. This is clear from 6.1.4 and the decomposition of $U_{1}$.

6.1.6. Theorem. (a) An L-distribution is on $U_{1}$ invertible iff its total mass is non-zero. in $\theta$.

(b) An 0-valued distribution on $U_{1}$ is invertible iff its total mass is a unit

Proof. Let $\mu$ be a distribution of $G^{\prime}$. By the above, it corresponds to a "polynomial"

$$
f\left(x_{1}, \ldots, x_{j}\right) .
$$

But $f(0, \ldots, 0)=$ total mass of $\mu$. Upon passing to the limit, the result follows.

As an example of a measure, we have the Dirac measure, $\delta_{x}, x \in X$. This is defined by

$$
\int_{X} f d \delta_{x}=f(x)
$$

Finally, one can talk about measures and distributions with values in $M(n, L)=\{n \times n$ matrices $\}$. We leave the details to the reader.

6.3. The $\pi$-adic theory. We now show how our $\pi$-adic functions arise out of $\pi$-adic distributions and measures. Fix $s=\left(s_{0}, s_{1}\right) \in S_{\infty}$, and let $X=\left\{\mathfrak{A}_{0}, \ldots, \mathfrak{A}_{m}\right\}$ be a collection of ideal class representatives. We use the notation of 2.3 .

Let $C$ be an integral ideal and $\mathscr{G}(C), \hat{g}(C)$, be as defined earlier, i.e., $\hat{g}(C)=\lim _{\tilde{m}} g(C) / P\left(\pi^{m} C\right)$. We begin by considering $\hat{g}(C)$ as a discrete 
group. The notion of a distribution on $\hat{g}(C)$ is obvious, if we agree to integrate only bounded locally constant functions.

Let $s=\left(s_{0}, s_{1}\right) \in S_{\infty}$ with $D\left(s_{0}\right)>0$.

6.2.1. Definition. We set $\mu_{s}=\Sigma_{(\mathscr{U}, C)=1 ; \mathfrak{A} \subseteq A} \mathfrak{U}^{-s} \delta_{\mathfrak{U}}$; where $\delta_{\mathfrak{U}}$ is the Dirac measure at $\mathfrak{A}$.

It is clear that $\mu_{s}$ is a distribution in the above sense.

Now $\mathscr{G}(C)$ (or rather its image) is dense in $\hat{\mathscr{G}}(C)$. Therefore, we can push this distribution forward to obtain one on $\hat{g}(C)$. We still denote this distribution by $\mu_{s}$.

6.2.2. Proposition. Let $\delta$ be a class of $\hat{g}(C) / \hat{P}\left(\pi^{m} C\right)=$ $g(C) / P\left(\pi^{m} C\right)$. Then,

$$
\mu_{s}(\delta)=\zeta(\delta, s)
$$

Proof. This follows immediately from the definition of the pullback of a distribution.

It is not hard to see $\mu_{s}(\delta)$ is a Laurent-polynomial, for $s=\left(s_{0},-i\right)$.

It is clear that we can now extend the definition of $\mu_{s}$ on $\hat{G}(C)$ to all $s \in S_{\infty}$. It is easy to see that $\mu_{s}$ is a measure for all $s \in S_{\infty}$, with $D\left(s_{0}\right) \geq 0$.

Let $D\left(s_{0}\right) \geq 0$.

6.2.3. LEMMA. Let $f$ be continuous on $\hat{g}(C)$. Then,

$$
\int_{\hat{G}(C)} f d \mu_{s} \text { is analytic in } s_{0}^{-1} \text {. }
$$

Proof. The integral is a uniform limit of analytic functions.

Recall now that in our set-up, $\langle\mathfrak{U}\rangle^{s_{1}}$ is thought of as being identified by some character on $\hat{g}(C)$, which induces $\langle n\rangle^{s_{1}}$ on $U_{1}$. If $x \in \hat{g}(C)$, we abuse notation and write $x^{s_{1}}$ for the value of this character. Then, we have

6.2.4. THEOREM. Let $D\left(s_{0}\right) \geq 0$. Then, if $s_{2} \in \mathbf{Z}_{p}$,

$$
\int_{\hat{q}_{(C)}} x^{-s_{2}} d \mu_{s}=\zeta(y) \prod_{\mathfrak{P} \mid C}\left(1-\mathfrak{B}^{-y}\right) ; \quad y=\left(s_{0}, s_{1}+s_{2}\right) .
$$

Proof. By our description of $\mu_{s}$ in terms of Dirac measures, this is obvious for $D\left(s_{0}\right)>0$. Thus, by analytic continuation, the result is immediate. 
In the same fashion, one can show

6.2.5. TheOREM. Let $f$ be a function on $G(C) / P\left(\pi^{m} C\right)$. Then, in the obvious meaning,

$$
\int_{\hat{g}_{(C)}} f x^{-s_{2}} d \mu_{s}=L(f, y) ; \quad y=\left(s_{0}, s_{1}+s_{2}\right) .
$$

6.2.6. Question. Does there exist a method by which we can extend the integration to all $\left(s_{0}, s_{1}\right)$ with $D\left(s_{0}\right)>-i, i \in \mathbf{N}$ arbitrary?

6.3. Group theoretic interpretation of zeros. 6.1 .6 allows us to give a distribution theoretic integration of the zeroes of our functions. We show how via an example: Let $C \subseteq A$ be an integral ideal and let $L(s)=$ $\left(\Pi_{\mathfrak{B} \mid C}\left(1-\mathfrak{B}^{-s}\right)\right) \zeta(s)$. Let $X=\left\{\mathfrak{U}_{0}, \ldots, \mathfrak{A}_{m}\right\},\left(\mathfrak{A}_{i}, C\right)=1$, all $i$.

We have just seen that

$$
\int_{\hat{q}(C)} d \mu_{s}=L(s)
$$

By using $X$, we can push our distribution onto $U_{1} \subseteq \hat{g}(C)$; it is defined over $K\left(s_{0}\right)$. Then 6.1 .6 implies

6.3.1. Proposition. $\mu_{s}$, considered on $U_{1}$, is not invertible as $K\left(s_{0}\right)$-distribution iff $L(s)=0$ conditionally.

6.4. The v-adic theory. We now mention the $v$-adic theory. As the proofs are exactly the same as for the $\pi$-adic case, we omit them.

Let $C$ be a fixed integral ideal, $\mathfrak{P}$ a prime, etc. We view $\hat{g}(C)_{v}$ as a discrete group and, for $v\left(x_{0}\right)>0$, we construct the distribution

$$
\sum_{\substack{(\mathfrak{A}, C)=1 \\ \mathfrak{U} \subseteq A}} X_{0}^{-D(\mathfrak{A})} \mathfrak{U}^{-x_{1}} \delta_{\mathfrak{A}}=\mu_{x} .
$$

As before, $\mu_{x}$ descends to a distribution $\hat{g}(C)_{v}$, which is still denoted $\mu_{x}$.

6.4.1. LEMMA. In the notation of 5.5.5, if $\delta \in \mathscr{S}(\mathfrak{P C} C) / P\left(\mathfrak{P}^{j} C\right)$, then

$$
\mu_{x}(\delta)=\zeta(\delta, x) \text {. }
$$

Thus, we can extend $\mu_{x}$ to all $x \in X_{v}$. It is a measure for $v\left(x_{0}\right) \geq 0$. 
6.5.1. TheOrem. Let $x_{2} \in \delta_{v}$. Then, if $v\left(x_{0}\right) \geq 0$,

$$
\int_{\hat{\mathfrak{g}}(C)} z^{-x_{2}} d \mu_{x}=\zeta_{v}(y) \prod_{\substack{\mathfrak{B} \mid C \\ \mathfrak{B} \neq \mathfrak{B} \\ \mathfrak{B} \text { prime }}}\left(1-\mathfrak{B}^{-y}\right), \quad y=\left(x_{0}, x_{1}+x_{2}\right) .
$$

We leave the corresponding result on characters to the reader.

One can also obtain distributions and measures on $\hat{g}\left(C \pi^{m}\right)_{v}$ in the above fashion. We also leave the (easy) details to the reader.

7. Galois group interpretation via class-field theory. We begin with a simple example. Let $A=\mathbf{F}_{r}[T]$ and let $\left(C_{\zeta}\right), \zeta \in \mathbf{F}_{r}^{*}$, be the rank one elliptic module given by

$$
C_{\zeta}(T)=T F^{0}-\zeta F
$$

Let $\pi=1 / T$; so "monicity" has its usual meaning. Let $I=(i)$ be an ideal and let $\alpha_{\zeta}$ be a primitive $I$ th division point in $\bar{k}$. It is an exercise to show that $k\left(\alpha_{\xi}\right) / k$ is Galois with group isomorphic to $(A / I)^{*}$ in the natural fashion and that $A\left[\alpha_{\zeta}\right]$ is the ring of integers.

Let $\mathfrak{P}=(P)$ be a prime not dividing $I$ and let $P$ be monic. It is trivial to see that

$$
C_{\zeta}(P)=(-\zeta)^{D(P)} F^{D(P)}+\{\text { lower terms whose coefficients are in }(P)\} .
$$

Thus, the Frobenius at $\mathfrak{P}$ is given by the image of $(-\zeta)^{-D(P)} P$ in $(A / I)^{*}$.

Let $\pi$ now be $-\zeta / T$. Then, via class-field theory, we see the Galois group of $k\left(\alpha_{s}\right) / k$ is $G(I) / P(I)$; where we use monics with respect to our new $\pi$.

We can now express $\zeta_{A\left[\alpha_{\xi}\right]}(s)$ in terms of $L$-series as in $\S 4$. From 5.4.5, we have

\subsection{THEOREM. $\zeta_{A\left[\alpha_{\zeta}\right]}(-i)=0$ for all $i \in \mathbf{N}$.}

As $A\left[\alpha_{\zeta}\right]$ is not "totally-real" (i.e., totally split at $\infty$ ) this result is in line with classical results.

We also obtain the fact that the ramification degree at $\infty$ is $(r-1)$. As $k\left(\alpha_{\zeta}^{r-1}\right)$ is the function field of $M_{I}^{1}$ and this splits totally at $\infty$, we see that $k\left(\alpha_{\zeta}\right) / k\left(\alpha_{\zeta}^{r-1}\right)$ is totally and tamely ramified above $\infty$. Thus, we have an explicit "cyclotomic" construction for the group $\mathscr{}(I) / P(I)$. This is due to D. Hayes following L. Carlitz; see ([1], [10]). It is the analog of the classical full cyclotomic field. 
Now let $A$ be general. It is easy to see that, via class-field theory, all groups of the form $G(C) / P\left(\pi^{m} C\right)$ occur as Galois groups of abelian extensions of $k$. A construction of the $A$-integers may be given in the same fashion as above, when $m=0$, by using the elliptic modules constructed in ([11]). Therefore, these extensions may also be thought of as being "cyclotomic". Further, the act of picking $\pi$ corresponds to choosing a totally ramified tower at $\infty$.

To finish, we now summarize what is known (see [8]), when $A=\mathbf{F}_{r}[T]$, $r=p^{n}$. Let $\mathfrak{B} \subseteq A$ be a prime and $k\left(\mathfrak{P}^{m}\right)$ be the field corresponding to $q(\mathfrak{B}) /\left(\mathfrak{B}^{m}\right)$. Let $D(\mathfrak{B})=d$. Let $h_{\mathfrak{P}^{m}}$ be the class number of this field and let $h_{\mathfrak{B}^{m}}^{+}$be the class number of the subfield fixed by $\mathbf{F}_{r}^{*}$. It it known that $h_{\mathfrak{B}^{m}}^{+} \mid h_{\mathfrak{P}^{m}}$ and we set $h_{\mathfrak{\beta}^{m}}^{-}=h_{\mathfrak{B}^{m}} / h_{\mathfrak{B}^{m}}^{+}$.

7.2. THEOREM. (a) $p \mid h_{\mathfrak{P}^{m}}^{-}$if and only if $\mathfrak{\Re} \mid \Pi_{i=1 ;(r-1)+i}^{r^{d}-2} \beta(i)$

(b) $p \mid h_{\mathfrak{B}^{m}}^{+}$if and only if $\mathfrak{B} \mid \prod_{i=1 ;(r-1) \mid i}^{r^{d}-2} \beta(i)$.

Now let $M \in A$, with $(M, \mathfrak{B})=1$ and $D(M)<d i$.

7.3. Definition. (a) We set

$$
\mu_{1}\left(M+\mathfrak{B}^{i}\right)=\left\{\begin{array}{ll}
2+(p) & \text { if } M \text { is monic } \\
1+(p) & \text { if } M \text { is non-monic }
\end{array}\right\} .
$$

(b) We set

$$
\mu_{2}\left(M+\mathfrak{P}^{i}\right)=\left\{\begin{array}{ll}
-D(M)+d i+(p) & \text { if } M \text { is monic } \\
-D\left(\mathfrak{P}^{i}\right)+(p) & \text { if } M \text { is non-monic. }
\end{array}\right\}
$$

Both measures have values in $\mathbf{F}_{p} \subseteq A$. The measure $\mu_{1}$ arises from $\S 6.4$ with $X=(1,0)$, and the measure $\mu_{2}$ arises by applying $d / d x_{0}$.

It is easy to use 6.5.1 and the vanishing of $\zeta(-i), i \equiv 0(r-1)$, to show the following

7.4. TheOrem. (a) Let $i \in \mathbf{N}^{+}$with $i \neq 0(r-1)$. Then

$$
\left(1-\mathfrak{P}^{i}\right) \beta(i)=\int_{A_{\mathfrak{*}}^{*}} x^{i} d \mu_{1}(x) .
$$

(b) Let $i \equiv 0(r-1)$. Then

$$
\left(1-\mathfrak{B}^{i}\right) \beta(i)=\int_{A_{\mathfrak{*}}^{*}} x^{i} d \mu_{2}(x) .
$$

Now let $P$ be the continuous function from $A_{\mathfrak{B}}^{*}$ to $A_{\mathfrak{P}}^{*}$ given by taking $x \rightarrow x^{-1}$. Let $\hat{\mu}_{i}=P_{*} \mu_{i}, i=1,2$. Let $\mathcal{C l}\left(\mathfrak{P}^{m}\right), \mathcal{C} \ell\left(\mathfrak{B}^{m}\right)_{+}$be the classgroups i.e., $\operatorname{Jac}\left(\mathbf{F}_{r}\right)$, of $k\left(\mathfrak{P}^{m}\right), k\left(\mathfrak{P}^{m}\right)_{+}$respectively. It then follows from 
the work of Tate and Gross that $\hat{\mu}_{1}$ annihilates $\mathcal{C l}\left(\mathfrak{B}^{m}\right) / p \mathcal{C l}\left(\mathfrak{P}^{m}\right)$ and $\hat{\mu}_{2}$ annihilates $\mathcal{C l}\left(\mathfrak{B}^{m}\right)_{+} / p \mathfrak{C l}\left(\mathfrak{P}^{m}\right)_{+}$.

Next, let $\zeta$ be a primitive $\left(r^{d}-1\right)$-st root of 1 and let $B=\mathbf{Z}_{p}[\zeta]$. Clearly $B$ is unramified over $\mathbf{Z}_{p}$ and $B / p B \simeq A / \mathfrak{B}$. If we fix one such isomorphism we obtain a character $\omega: A / \mathfrak{P}^{*} \rightarrow B^{*}$. All characters are powers of $\omega$ and, upon tensoring with $B$, we can form isotopic components $\mathcal{C}\left(\mathfrak{P}^{m}\right)\left(\omega^{i}\right)$, in the usual cyclotomic fashion. Putting all this together we finally have

7.5. TheOREM. Let $0<i<r^{d}-1$. Then $\mathcal{C l}\left(\mathfrak{P}^{m}\right)\left(\omega^{i}\right) \neq 0$ implies that $\mathfrak{B} \mid \beta\left(r^{d}-1-i\right)$.

As it is trivial to see, from its additive expansion, that $\beta(p i)=\beta(i)^{p}$, the result is invariant of the choice of $\omega$.

7.6. Question. What is the meaning of the Bernoulli-Carlitz numbers?

(Note: This pattern also seems to hold with $r=2$ and $r=4$. With $r=4$ the numbers differ by powers of 4 !)

\section{REFERENCES}

1. L. Carlitz, $A$ class of polynomials, Trans. Amer. Math. Soc., 43 (1938), 168-182.

2. V. G. Drinfeld, Elliptic modules, (English Translation), Math. USSR, Sbornik, 23 (1974).

3. __ Elliptic modules II, Math. USSR, Sbornik, 31 (1977), No. 2.

4. S. Galovich, M. Rosen, The class number of cyclotomic function fields, J. Number Theory, 13, No. 3, (1981), 363-375.

5. D. Goss, The algebraist's upper half-plane, Bull. Amer. Math. Soc., 2, \#3, (May, 1980), 391-415.

$6 . \quad$ v-adic zeta functions, L-series and measures for function fields, Inventiones Mathematicae, 55 (1979), 107-119.

7. __ The T-ideal and special zeta-values, Duke Math. J., (June, 1980), 345-364.

8. Kummer and Herbrand criterion in the theory of function fields, Duke Math. J., 49 (1982), 377-384.

9. On a Fermat equation arising in the arithmetic theory of function fields, (to appear in Math. Annalen).

10. D. Hayes, Explicit class field theory for rational function fields, Trans. Amer. Math. Soc., 189 (1979), 77-91.

11. Explicit class field theory in global function fields, Studies in Algebra and Number Theory, G.-C. Rota (ed.), Academic Press, (1979).

12. L. I. Wade, Certain quantities transcendental over $G F\left(p^{n}, x\right)$, Duke Math. J., 8 (1941), 701-720.

Received October 10, 1980. 



\section{PACIFIC JOURNAL OF MATHEMATICS}

EDITORS

Donald BABBITT (Managing Editor)

University of California

Los Angeles, CA 90024

Hugo Rossi

University of Utah

Salt Lake City, UT 84112

C. C. Moore and Arthur Ogus

University of California

Berkeley, CA 94720
J. DugunduI

Department of Mathematics

University of Southern California

Los Angeles, CA 90089-1113

R. FinN and H. SAmelson

Stanford University

Stanford, CA 94305

\section{ASSOCIATE EDITORS}
R. ARENS
E. F. BECKENBACH
B. H. NEUMANN
F. WolF
K. YoshidA (1906-1982)

\section{SUPPORTING INSTITUTIONS}

UNIVERSITY OF ARIZONA

UNIVERSITY OF BRITISH COLUMBIA

CALIFORNIA INSTITUTE OF TECHNOLOGY

UNIVERSITY OF CALIFORNIA

MONTANA STATE UNIVERSITY

UNIVERSITY OF NEVADA, RENO

NEW MEXICO STATE UNIVERSITY

OREGON STATE UNIVERSITY
UNIVERSITY OF OREGON

UNIVERSITY OF SOUTHERN CALIFORNIA

STANFORD UNIVERSITY

UNIVERSITY OF HAWAII

UNIVERSITY OF TOKYO

UNIVERSITY OF UTAH

WASHINGTON STATE UNIVERSITY

UNIVERSITY OF WASHINGTON 


\section{Pacific Journal of Mathematics}

Vol. 105, No. 1 September, 1983

Kenneth F. Andersen, On the transformation of Fourier coefficients of

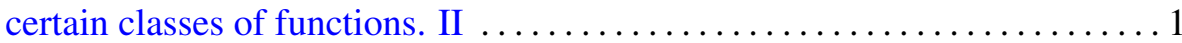

Gavin Brown, Irving Leonard Glicksberg and Edwin Hewitt, Indicator functions with large Fourier transforms $\ldots \ldots \ldots \ldots \ldots \ldots \ldots \ldots \ldots \ldots$

Shih-Sen Chang, Some random fixed point theorems for continuous random

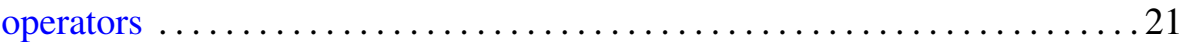

K. C. Chattopadhyay and Olav Njstad, Quasiregular nearness spaces and

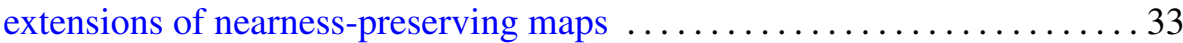

Thomas W. Cusick, The two-dimensional Diophantine approximation

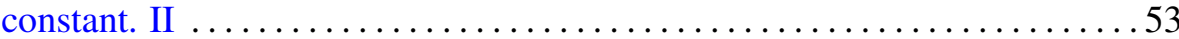

Eric Karel van Douwen and Jan van Mill, Spaces without remote points . . .669 Hector O. Fattorini, Convergence and approximation theorems for

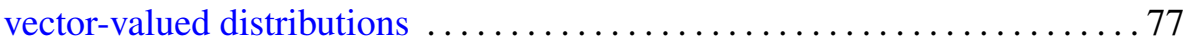

John J. F. Fournier and Louis Pigno, Analytic and arithmetic properties of

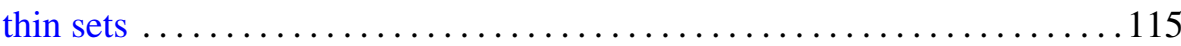

David Goss, On a new type of $L$-function for algebraic curves over finite

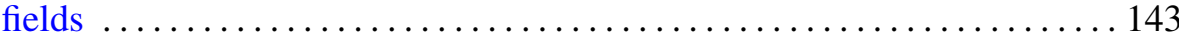

Douglas Austin Hensley, Lattice vertex polytopes with interior lattice

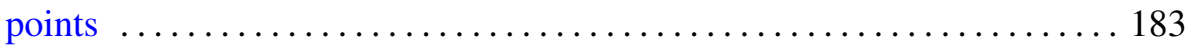

J. K. Kohli, Monotone extensions of mappings and their applications ...... 193

John C. Morgan, II, On equivalent category bases . . . . . . . . . . . . 207

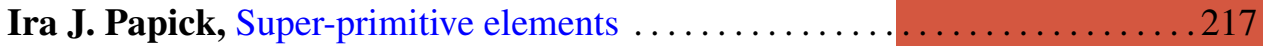

José Luis Rubio de Francia and José Luis Torrea, Vector extensions of

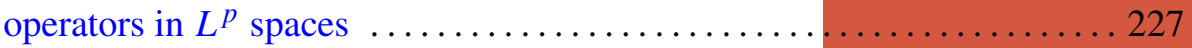

Mark Phillip Thomas, Closed ideals of $l^{1}\left(\omega_{n}\right)$ when $\left\{\omega_{n}\right\}$ is star-shaped $\ldots 237$ 